NOTICE: this is the author's version of a work that was accepted for publication in Mechanical Systems and Signal Processing. Changes resulting from the publishing process, such as peer review, editing, corrections, structural formatting, and other quality control mechanisms may not be reflected in this document. Changes may have been made to this work since it was submitted for publication. A definitive version was subsequently published in Mechanical Systems and Signal Processing, Vol. 36, No. 1 (2013).

DOI: 10.1016/j.ymssp.2011.07.010 


\title{
Analytical and Experimental Comparisons of Electromechanical Vibration Response of a Piezoelectric Bimorph Beam for Power Harvesting
}

\author{
M. F. Lumentut, I. M. Howard \\ Theoretical and Applied Mechanics, Department of Mechanical Engineering \\ Curtin University, Australia
}

\begin{abstract}
Power harvesters that extract energy from vibrating systems via piezoelectric transduction show strong potential for powering smart wireless sensor devices in applications of health condition monitoring of rotating machinery and structures. This paper presents an analytical method for modelling an electromechanical piezoelectric bimorph beam with tip mass under two input base transverse and longitudinal excitations. The Euler-Bernoulli beam equations were used to model the piezoelectric bimorph beam. The polarity-electric field of the piezoelectric element is excited by the strain field caused by base input excitation, resulting in electrical charge. The governing electromechanical dynamic equations were derived analytically using the weak form of the Hamiltonian principle to obtain the constitutive equations. Three constitutive electromechanical dynamic equations based on independent coefficients of virtual displacement vectors were formulated and then further modelled using the normalised Ritz eigenfunction series. The electromechanical formulations include both the series and parallel connections of the piezoelectric bimorph. The multi-mode frequency response functions (FRFs) under varying electrical load resistance were formulated using Laplace transformation for the multi-input mechanical vibrations to provide the multi-output dynamic displacement, velocity, voltage, current and power. The experimental and theoretical validations, reduced for the single mode system were shown to provide reasonable predictions. The model results from polar base excitation for off-axis input motions were validated with experimental results showing the change to the electrical power frequency response amplitude as a function of excitation angle, with relevance for practical implementation.
\end{abstract}

Keyword : Electromechanical, piezoelectric, harvesting, Laplace, Hamiltonian, Weak form. 


\section{Introduction}

The development of permanent embedded computing-based equipment has increased the demand from engineering industry to monitor or diagnose the health condition of structures and rotating machinery. The prevalent technological equipment still requires electrical power from the mains power supply or battery in order to read and transfer the electrical data signals via wireless sensor nodes into computer networks including data acquisition, instrument control and/or analyzers for condition health monitoring. An example in-depth theoretical and experimental review of such diagnosis and prognosis in the area of defence technology includes bearing vibration monitoring for engine turbomachinery as discussed by Howard [1]. The conventional battery systems have a limited lifespan for power production. The systems are still dependent upon the electrical power from the battery or mains power supply for recharging. This leads to the tedious task of replacing the conventional battery from the smart sensor device often located in remote or inaccessible areas. Machinery vibration presents an ideal application for piezoelectric power harvesting providing continuous power to sensor networks whenever the equipment is operating. An example for possible future development can be found in the application of aircraft sensor network systems to tackle self-diagnosis of engines and structures, [2].

The application of power harvesting using cantilevered piezoelectric beams under input dynamic motion coupling with power electronic components has been an attractive field to be investigated both mathematically and experimentally by researchers. The investigation of a single mode of the mathematical model for scavenging low electrical power based on a range of frequency responses using the piezoelectric-based accelerometer and the cantilever piezoelectric models under input transverse base motion has also become a topic of interest. The electrical equivalent representation of the electromechanical piezoelectric structure has been discussed by Roundy and Wright [3], investigating the single mode of the piezoelectric beam with two different sizes of tip mass to show the trend of electrical voltage. Later work from duToit et al $[4,5]$ investigated a single mode of the mathematical models for scavenging low electrical power based on a range of frequency responses using the lumped-mass piezoelectric-based accelerometer model and the cantilever piezoelectric model using Rayleigh-Ritz's method under input transverse base motion. The strain resulting from mechanical vibration associated with piezoelectric modes of operations of the cantilevered piezoelectric beam structure was shown to directly affect the electrical power output during 
the dynamic response. In the analytical solution, the short and open circuit models of power harvesting were optimised to obtain power harvesting based on the frequency response under various load impedances. The comparison between the experiment and theory was also undertaken. However, the power at the resonance region seemed to under predict the results. Kim et al [6] further discussed the vibration energy harvester performance by considering the effects of tip mass geometry on the bimorph. The single mode of the electromechanical dynamic equations in scalar form given from duToit's representation [4], was modelled based on the Rayleigh-Ritz's method. The trends of tip transverse displacement, voltage and power harvesting with and without tip masses were plotted with respect to the variation of load resistances where slight difference of results between the model and experiment were found. Although, the purpose of simulation was the single mode FRFs, the multi-mode FRF model can provide a much more accurate representation as it can be adjusted to show the single mode response.

The closed-form analytical model for a vibration power harvester using the cantilever piezoelectric beam under input transverse excitation has been investigated using the normalised eigenfunction form [7]. The constitutive electromechanical equations showed the frequency response analysis of the tip transverse displacement, voltage and power modelled under varying load resistance. The frequency response electrical voltage and power analytical results showed good agreement with the experimental measurements. Goldschmidtboeing and Woias [8] investigated different shaped rectangle and truncated triangular piezoelectric beams with varying tip mass under base transverse excitation using Ritz-Rayleigh's method. They showed that varying the mass ratio between tip mass and piezoelectric mass and truncated ratio or shape ratio between the rectangle and triangular portion of the piezoelectric beam could be used for optimum power tuning. They noticed that the triangular shaped beam provided greater power when compared with the rectangular beam. Shu and Lien [10] discussed a cantilevered bimorph piezoelectric beam coupled with an electronic circuit under dynamic input force. They provided an analytical model to obtain the non-dimensional normalized parameters of displacement, voltage and electrical power where the formulations were used to obtain the optimal parameter functions. Renaud et al [9] discussed the unimorph piezoelectric beam under input impact load to generate the electric voltage. The impact load was from a slider that hit the tip of the piezoelectric element. The electrical equivalent method was used to analyse the coupled unimorph electrical and mechanical system using the lumped mass single degree of freedom model. Recent power harvesting research using a new 
piezoelectric material, the single crystal relaxor ferroelectric material (PMN-PT) has been investigated by Mathers et al [11], where the fabricated micro-piezoelectric cantilever beam with proof mass was used for predicting the vibration power harvesting. The use of piezoelectric material from PMN-PT with the interdigitated electrode (IDE) was aimed to improve the energy conversion efficiency where the use of varying proof mass from the polydimethylsiloxane (PDMS) aimed to tune the natural frequency. The analytical model of the elastic vibrating beam associated with the direct effect of the piezoelectric equation was used to give the electrical voltage frequency response. However, the modelled electromechanical dynamic behaviour of the piezoelectric beam did not consider the effect of backward piezoelectric coupling on the power harvesting model for the frequency responses of displacement, voltage and power.

Smart structures and associated mathematical modeling has been an attractive field for many researchers with various applications, although the previous references have not clearly provided the analytical methods in terms of Hamiltonian mechanics. In the earlier mathematical concepts, $[12,13]$ the piezoelectric crystal plate equations were derived using variational calculus establishing the constitutive dynamic equations of the electromechanical components. Later on, the application areas were extended to control systems where this included the analytical methods for control of the bimorph vibration [14] and the usage of piezoelectric tubes subjected to periodic excitation [15] using Hamiltonian mechanics. Some recent developments of piezoelectric technology concern the usage of piezoelectric material placed as a patch onto the structure and subject to ambient mechanical vibration in order to convert the vibration to useful electrical energy. Recent mathematical study of the weak and closed forms of the electromechanical dynamic equations of the piezoelectric bimorph with tip mass [16, 17] were derived using the strong form of Hamiltonian's principle. The normalised Ritz eigenfunction form was used to further formulate the weak form method whereas normalised direct analytical solution using boundary value method was further used to formulate the closed form method. The Laplace transformation in terms of frequency response functions were then applied to the electromechanical dynamic equations in order to give multi-output for multi-input responses. The validation with the experimental results in $[17,18]$ was also given with good agreement by considering one and two input base motions on the piezoelectric bimorph.

This paper presents a novel analytical model of the dynamic behaviour of an electromechanical piezoelectric bimorph beam based on the normalised Ritz method using 
the weak form of Hamiltonian's principle. The Laplace transform of the dynamic equations based on the strain-polarity-electric field effects of the piezoelectric bimorph under two input base transverse and longitudinal excitations has been used to show the multi-mode frequency response functions. This includes the effect of input base transverse and longitudinal excitations onto the bimorph, where the parametric electromechanical effects of the piezoelectric bimorph due to the input base excitation affected the existence of the strain fields. It is also noted that the electrical force and moment of the piezoelectric bimorph can be further extended to establish the forward and backward piezoelectric couplings due to the effect of the transverse and longitudinal stress field of the piezoelectric bimorph interlayer which can affect the mechanical and electrical dynamic behaviours. Moreover, the theoretical analysis and experimental results of the electromechanical bimorph frequency response functions (FRFs) under the input base transverse acceleration were validated using measurement of the tip absolute dynamic displacement, velocity, electrical voltage, current and power harvesting. In addition, the FRFs of the bimorph with the tip mass under the action of simultaneous transverse and longitudinal accelerations are analysed to show polar power harvesting results.

\section{Mathematical Analysis}

The constitutive electromechanical dynamic equations of the piezoelectric bimorph beam was formulated using the weak form of Hamiltonian theorem that consisted of the strain energy of the central bimorph substructure (brass shim), the linear electrical enthalpy for the upper and lower piezoelectric layers, and the kinetic energy of the bimorph including the tip mass. The application of typical PZT material was considered in the theoretical bimorph beam model using the plane-stress relationship, the $\{3-1\}$ mode of operation and the induced electrical field was developed in the z-direction or thickness of the material. The cantilevered piezoelectric bimorph beam was considered to have simultaneous two input base transverse and longitudinal excitations. The constitutive dynamic equation after simplifying $[16,17]$ can be written as,

$$
\begin{aligned}
& \int_{t_{1}}^{t_{2}}\left[\int _ { \Omega } \left(C_{11}^{(D, k)} \frac{\partial u_{r e l}}{\partial x} \frac{\partial \delta u_{r e l}}{\partial x}+C_{11}^{(F, k)} \frac{\partial^{2} w_{r e l}}{\partial x^{2}} \frac{\partial^{2} \delta w_{r e l}}{\partial x^{2}}-R_{31}^{(G, k)} v \frac{\partial \delta u_{r e l}}{\partial x}+R_{31}^{(H, k)} v \frac{\partial^{2} \delta w_{r e l}}{\partial x^{2}}+I^{(A, k)} \ddot{u}_{r e l} \delta u_{r e l}\right.\right. \\
& -S_{33}^{(k)} v(t) \delta v(t)+I^{(C, k)} \frac{\partial \ddot{w}_{r e l}}{\partial x} \frac{\partial \delta\left(w_{r e l}\right)}{\partial x}+I^{(A, k)} \ddot{w}_{r e l} \delta w_{r e l}-R_{31}^{(G, k)} \frac{\partial u_{r e l}}{\partial x} \delta v
\end{aligned}
$$




$$
\begin{aligned}
& \left.+R_{31}^{(H, k)} \frac{\partial^{2} w_{r e l}}{\partial x^{2}} \delta v+I^{(A, k)} \ddot{u}_{\text {base }} \delta u_{r e l}+I^{(A, k)} \ddot{w}_{b a s e} \delta w_{r e l}\right) \mathrm{d} x \mathrm{~d} y-q \delta v+I_{\text {tip }}^{(A)} \ddot{u}_{r e l}(L) \delta u_{r e l}(L) \\
& +I_{\text {tip }}^{(C)} \frac{\partial \ddot{w}_{r e l}}{\partial x}(L) \frac{\partial \delta w_{r e l}}{\partial x}(L)+I_{\text {tip }}^{(A)} \ddot{u}_{\text {base }} \delta u_{r e l}(L)+I_{\text {tip }}^{(A)} \ddot{w}_{b a s e} \delta w_{r e l}(L)+I_{\text {tip }}^{(A)} \ddot{w}_{r e l}(L) \delta w_{r e l}(L) \\
& \left.+\oint_{S}\left(-n_{x} N_{x x}^{(D, k)} \delta u_{r e l}+n_{x} \frac{\partial M_{x x}^{(F, k)}}{\partial x} \delta w_{r e l}-n_{x} M_{x x}^{(F, k)} \frac{\partial \delta w_{r e l}}{\partial x}-I^{(C, k)} n_{x} \frac{\partial \ddot{w}_{r e l}}{\partial x} \delta w_{r e l}\right) \mathrm{d} S\right] \mathrm{d} t=0
\end{aligned}
$$

The variables $u_{r e l}, w_{r e l}, v, q, u_{\text {base }}$ and $w_{\text {base }}$ indicate the relative longitudinal and transverse displacement fields, voltage, electrical charge and input base longitudinal and transverse excitations, respectively. Moreover, the coefficients $C_{11}^{(D, k)}, C_{11}^{(F, k)}, R_{31}^{(G, k)}, R_{31}^{(H, k)}$, $S_{33}^{(k)}, I^{(A, k)}, I^{(C, k)}, I_{\text {tip }}^{(A)}, I_{\text {tip }}^{(C)}, N_{x x}^{(D, k)}$ and $M_{X X}^{(F, k)}$ indicate the longitudinal extension and transverse stiffness coefficients reduced from plane stress, longitudinal extension and transverse piezoelectric couplings, capacitance of the piezoelectric element, zeroth and second mass moment of inertia of the bimorph, zeroth and second mass moment of inertia of the tip mass, and in-plane force and moment of the bimorph, respectively. Each coefficient from Eq. (1) is given in more detail in appendices A, B and C. Superscript $k$ indicates the layers of the bimorph. It should be noted that the second integral represents the divergence theorem reflecting the boundary conditions over the surface $S$ of the bimorph element in the direction $n_{x}$ of the unit vector normal to the $x$-axis. The second integral from Eq. (1) is sometimes called the generalised internal force and moment for every element discretisation and these become necessary when the element boundary $S$ coincides with boundary of domain $\Omega$ and their existence depends on external loads on certain nodes of the structure. The second integral can be a crucial part to be included when using finite element analysis if external loads are applied to the structure. In terms of the analytical approach proposed here, the second integral can be ignored because the displacement fields $\left(u_{r e l}, w_{r e l}\right)$ and virtual displacement fields $\left(\delta u_{r e l}, \delta w_{r e l}\right)$ were assumed as eigenfunction forms which meet the continuity of mechanical form or strain field and boundary geometry.

The solutions of Eq. (1) can be obtained using eigenfunction series of longitudinal extension and transverse bending effects. The form of the solutions can be prescribed as,

$$
w_{r e l}(x, t)=\sum_{r=1}^{m} w_{r}(t) \Psi_{r}(x) \quad, \quad u_{r e l}(x, t)=\sum_{r=1}^{m} u_{r}(t) \Theta_{r}(x)
$$


where parameters, $\Psi_{r}(x)$ and $\Theta_{r}(x)$ are defined as the independent mode shapes of relative motions in the form of eigenfunction series. In this case, these parameters can be determined using analytical solution forms for the cantilevered piezoelectric beam with a tip mass which can be formulated as shown in appendix D. Corresponding to Eq. (2), Eq. (1) can be further formulated in terms of eigenfunction forms by setting virtual displacement forms $\delta u_{r}(t)$, $\delta w_{r}(t)$ and $\delta v(t)$ separately to obtain three independent dynamic equations using the variational principle. Parameters of virtual displacements meet the duBois-Reymond's lemma to indicate that only dynamic equations have solutions. At this point, three dynamic equations of the piezoelectric bimorph beam can be formulated.

The first dynamic equation represents the electromechanical piezoelectric bimorph under longitudinal extension. Here it is written as,

$$
\begin{gathered}
\sum_{q=1}^{m}\left\{\sum_{r=1}^{m}\left[\int_{\Omega} I^{(A, k)} \Theta_{q}(x) \Theta_{r}(x) \ddot{u}_{r}(t) \mathrm{d} x \mathrm{~d} y+I_{\text {tip }}^{(A)} \Theta_{q}(L) \Theta_{r}(L) \ddot{u}_{r}(t)+\int_{\Omega} C_{11}^{(D, k)} \frac{\mathrm{d} \Theta_{q}(x)}{\mathrm{d} x} \frac{\mathrm{d} \Theta_{r}(x)}{\mathrm{d} x} u_{r}(t) \mathrm{d} x \mathrm{~d} y\right]\right. \\
\left.-\int_{\Omega} R_{31}^{(G, k)} \frac{\mathrm{d} \Theta_{q}(x)}{\mathrm{d} x} v(t) \mathrm{d} x \mathrm{~d} y+\int_{\Omega} I^{(A, k)} \Theta_{q}(x) \ddot{u}_{\text {base }}(t) \mathrm{d} x \mathrm{~d} y+I_{\text {tip }}^{(A)} \Theta_{q}(L) \ddot{u}_{\text {base }}(t)\right\} \delta u_{q}(t)=0
\end{gathered}
$$

The second dynamic equation represents the electromechanical piezoelectric bimorph under transverse bending form. It can be stated as,

$$
\begin{aligned}
& \sum_{q=1}^{m}\left\{\sum _ { r = 1 } ^ { m } \left[\int_{\Omega} I^{(A, k)} \Psi_{q}(x) \Psi_{r}(x) \ddot{w}_{r}(t) \mathrm{d} x \mathrm{~d} y+\int_{\Omega} I^{(C, k)} \frac{\mathrm{d} \Psi_{q}(x)}{\mathrm{d} x} \frac{\mathrm{d} \Psi_{r}(x)}{\mathrm{d} x} \ddot{w}_{r}(t) \mathrm{d} x \mathrm{~d} y+I_{\text {tip }}^{(A)} \Psi_{q}(L) \Psi_{r}(L) \ddot{w}_{r}(t)\right.\right. \\
& \left.+I_{\text {tip }}^{(C)} \frac{\mathrm{d} \Psi_{q}(L)}{\mathrm{d} x} \frac{\mathrm{d} \Psi_{r}(L)}{\mathrm{d} x} \ddot{w}_{r}(t)+\int_{\Omega} C^{(F, k)} \frac{\mathrm{d}^{2} \Psi_{q}(x)}{\mathrm{d} x^{2}} \frac{\mathrm{d}^{2} \Psi_{r}(x)}{\mathrm{d} x^{2}} w_{r}(t) \mathrm{d} x \mathrm{~d} y\right]+\int_{\Omega} R_{31}^{(H, k)} \frac{\mathrm{d}^{2} \Psi_{q}}{\mathrm{~d} x^{2}} v(t) \mathrm{d} x \mathrm{~d} y \\
& \left.\quad+\int_{\Omega} I^{(A, k)} \Psi_{q}(x) \ddot{w}_{\text {base }}(t) \mathrm{d} x \mathrm{~d} y+I_{\text {tip }}^{(A)} \Psi_{q}(L) \ddot{w}_{\text {base }}(t)\right\} \delta w_{q}(t)=0
\end{aligned}
$$

The third dynamic equation represents the electromechanical piezoelectric bimorph under electrical form. It can be written as,

$$
\sum_{r=1}^{m} \int_{\Omega}\left[-R_{31}^{(G, k)} \frac{\mathrm{d} \Theta_{r}(x)}{\mathrm{d} x} u_{r}(t)+R_{31}^{(H, k)} \frac{\mathrm{d}^{2} \Psi_{r}(x)}{\mathrm{d} x^{2}} w_{r}(t)\right] \mathrm{d} x \mathrm{~d} y \delta v(t)-\int_{\Omega} S_{33}^{(k)} v(t) \delta v(t) \mathrm{d} x \mathrm{~d} y=0
$$

or it can be differentiated with respect to time to obtain current and with the addition of an external resistor into Eq. (5) to give,

$$
-\sum_{r=1}^{m} \int_{\Omega} R_{31}^{(G, k)} \frac{\mathrm{d} \Theta_{r}(x)}{\mathrm{d} x} \dot{u}_{r}(t) \mathrm{d} x \mathrm{~d} y+\sum_{r=1 \Omega}^{m} \int_{31} R_{31}^{(H, k)} \frac{\mathrm{d}^{2} \Psi_{r}(x)}{\mathrm{d} x^{2}} \dot{w}_{r}(t) \mathrm{d} x \mathrm{~d} y-\int_{\Omega} S_{33}^{(k)} \dot{v}(t) \mathrm{d} x \mathrm{~d} y-\frac{1}{R_{\text {load }}} v(t)=0
$$


The constitutive dynamic equations from Eqs. (3), (4) and (6) can be reformulated in matrix form by including the mechanical damping coefficients after integration with respect to $y$ to give,

$$
\left[\begin{array}{ccc}
M_{q r}^{(u)} & 0 & 0 \\
0 & M_{q r}^{(w)} & 0 \\
0 & 0 & 0
\end{array}\right]\left\{\begin{array}{l}
\ddot{u}_{r} \\
\ddot{w}_{r} \\
\ddot{v}
\end{array}\right\}+\left[\begin{array}{ccc}
C_{q r}^{(u)} & 0 & 0 \\
0 & C_{q r}^{(w)} & 0 \\
P_{r}^{(u)} & P_{r}^{(w)} & P_{D}
\end{array}\right]\left\{\begin{array}{l}
\dot{u}_{r} \\
\dot{w}_{r} \\
\dot{v}
\end{array}\right\}+\left[\begin{array}{ccc}
K_{q r}^{(u)} & 0 & P_{q}^{(u)} \\
0 & K_{q r}^{(w)} & P_{q}^{(w)} \\
0 & 0 & R_{L}
\end{array}\right]\left\{\begin{array}{l}
u_{r} \\
w_{r} \\
v
\end{array}\right\}=\left[\begin{array}{ccc}
-Q_{q}^{(u)} & 0 & 0 \\
0 & -Q_{q}^{(w)} & 0 \\
0 & 0 & 0
\end{array}\right]\left\{\begin{array}{l}
\ddot{u}_{\text {base }} \\
\ddot{w}_{\text {base }} \\
\ddot{v}_{\text {base }}
\end{array}\right\}
$$

where:

$$
\begin{gathered}
M_{q r}^{(u)}=\int_{0}^{L} \hat{I}^{(A, k)} \Theta_{q}(x) \Theta_{r}(x) \mathrm{d} x+I_{\text {tip }}^{(A)} \Theta_{q}(L) \Theta_{r}(L) \\
M_{q r}^{(w)}=\int_{0}^{L} \hat{I}^{(A, k)} \Psi_{q}(x) \Psi_{r}(x) \mathrm{d} x+\int_{0}^{L} \hat{I}^{(C, k)} \frac{\mathrm{d} \Psi_{q}(x)}{\mathrm{d} x} \frac{\mathrm{d} \Psi_{r}(x)}{\mathrm{d} x} \mathrm{~d} x+I_{\text {tip }}^{(A)} \Psi_{q}(L) \Psi_{r}(L)+I_{\text {tip }}^{(C)} \frac{\mathrm{d} \Psi_{q}}{\mathrm{~d} x}(L) \frac{\mathrm{d} \Psi_{r}}{\mathrm{~d} x}(L) \\
K_{q r}^{(u)}=\int_{0}^{L} \hat{C}_{11}^{(D, k)} \frac{\mathrm{d} \Theta_{q}(x)}{\mathrm{d} x} \frac{\mathrm{d} \Theta_{r}(x)}{\mathrm{d} x} \mathrm{~d} x \quad, \quad K_{q r}^{(w)}=\int_{0}^{L} \hat{C}_{11}^{(F, k)} \frac{\mathrm{d}^{2} \Psi_{q}(x)}{\mathrm{d} x^{2}} \frac{\mathrm{d}^{2} \Psi_{r}(x)}{\mathrm{d} x^{2}} \mathrm{~d} x \\
P_{r}^{(u)}=-\int_{0}^{L} \hat{R}_{31}^{(G, k)} \frac{\mathrm{d} \Theta_{r}(x)}{\mathrm{d} x} \mathrm{~d} x, P_{r}^{(w)}=\int_{0}^{L} \hat{R}_{31}^{(H, k)} \frac{\mathrm{d}^{2} \Psi_{r}(x)}{\mathrm{d} x^{2}} \mathrm{~d} x, R_{L}=-\frac{1}{R_{l o a d}}, P_{D}=-\int_{0}^{L} \hat{S}^{(k)} \mathrm{d} x \\
Q_{q}^{(u)}=\int_{0}^{L} \hat{I}^{(A, k)} \Theta_{q}(x) \mathrm{d} x+I_{t i p}^{(A, k)} \Theta_{q}(L), Q_{q}^{(w)}=\int_{0}^{L} \hat{I}^{(A, k)} \Psi \Psi_{q}(x) \mathrm{d} x+I_{t i p}^{(A)} \Psi_{q}(L) \\
C_{q r}^{(u)}=\alpha_{u} M_{q r}^{(u)}+\beta_{u} K_{q r}^{(u)}, C_{q r}^{(w)}=\alpha_{w} M_{q r}^{(w)}+\beta_{w} K_{q r}^{(u)}, \hat{I}^{(A, k)}=b I^{(A, k)}, \hat{I}^{(C, k)}=b I^{(C, k)} \\
\hat{C}_{11}^{(D, k)}=b C_{11}^{(D, k)}, \hat{C}_{11}^{(F, k)}=b C_{11}^{(F, k)}, \hat{R}_{31}^{(G, k)}=b R_{31}^{(G, k)}, \quad \hat{R}_{31}^{(H, k)}=b R_{31}^{(H, k)}, \hat{S}_{33}^{(k)}=b S_{33}^{(k)}
\end{gathered}
$$

It should be noted that the $\wedge$ symbol refers to the modified variables after multiplying with the width $b$ of the bimorph. Eq. (7) represents the non homogeneous differential dynamic equation of the piezoelectric bimorph beam with two input base excitations after considering the rotary inertias of the bimorph and tip mass. In this case, we ignored the second part from $M_{q r}^{(w)}$ which refers to the rotary inertia of the bimorph component. Eq. (7) can also be used for modelling the piezoelectric bimorph using either series or parallel electrical connection. The connections just depend on the chosen piezoelectric couplings and also the chosen internal capacitance which will be considered in the next section. In addition to that, other parameters from this case such as mass moment of inertia, stiffness coefficients, piezoelectric constant and permittivity are viewed as constant values. The analysis must also consider the geometry of the piezoelectric bimorph where it will affect all aspects of power harvesting performance. The geometry of the piezoelectric bimorph beam with the tip mass was modelled as shown in 
Fig. 1. Variables $L, h_{s}$ and $h_{p}$ indicate the bimorph length, substructure thickness and piezoelectric thickness (same thickness between bottom and top layers), respectively. Other geometry parameters of tip mass can be found in Table 1 .

\subsection{Normalised Constitutive Electromechanical Dynamic Equations}

Corresponding with the convergent eigenfunction forms of Eq. (2), Eqs. (3), (4) and (5) need to be modified in order to achieve the orthonormality conditions. In this case, we introduce the convergent space- and time-dependent Ritz eigenfunction forms as,

$$
w_{r e l}(x, t)=\sum_{r=1}^{m} c_{r}^{(w)} \Psi_{r}(x) e^{i \omega t} \quad, \quad u_{r e l}(x, t)=\sum_{r=1}^{m} c_{r}^{(u)} \Theta_{r}(x) e^{i \omega t}
$$

In terms of considering only the mechanical equation, Eq. (8) can be substituted into Eqs. (3) and (4) to give the independent algebraic equations of the eigenvalues corresponding to the longitudinal and transverse bending form as,

$$
\begin{aligned}
& \sum_{r=1}^{m}\left[K_{q r}^{(u)}-\omega^{(u)^{2}} M_{q r}^{(u)}\right] c_{r}^{(u)}=0, \quad q=1,2, \ldots . ., m \\
& \sum_{r=1}^{m}\left[K_{q r}^{(w)}-\omega^{(w)^{2}} M_{q r}^{(w)}\right] c_{r}^{(w)}=0, \quad q=1,2, \ldots ., m
\end{aligned}
$$

or Eqs. (9) and (10) can be respectively formulated into explicit forms as,

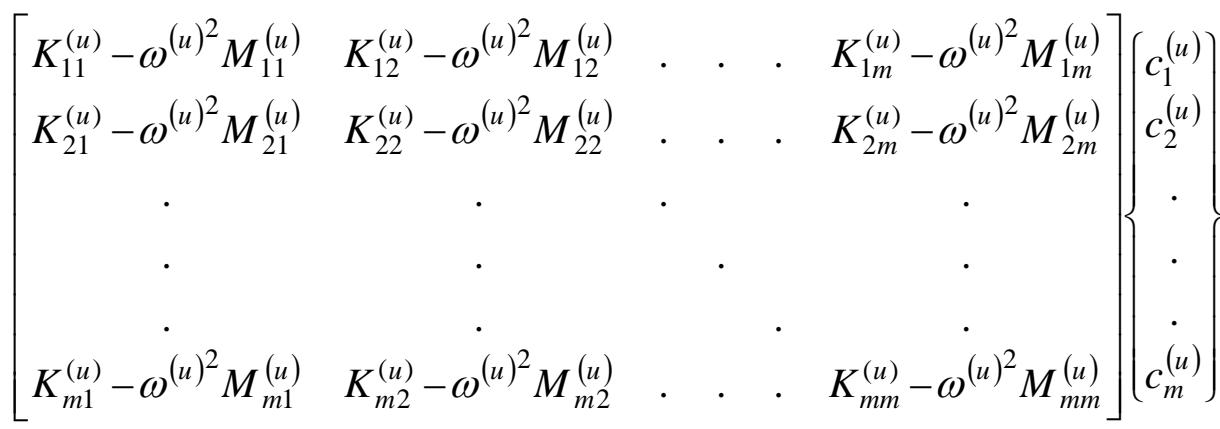

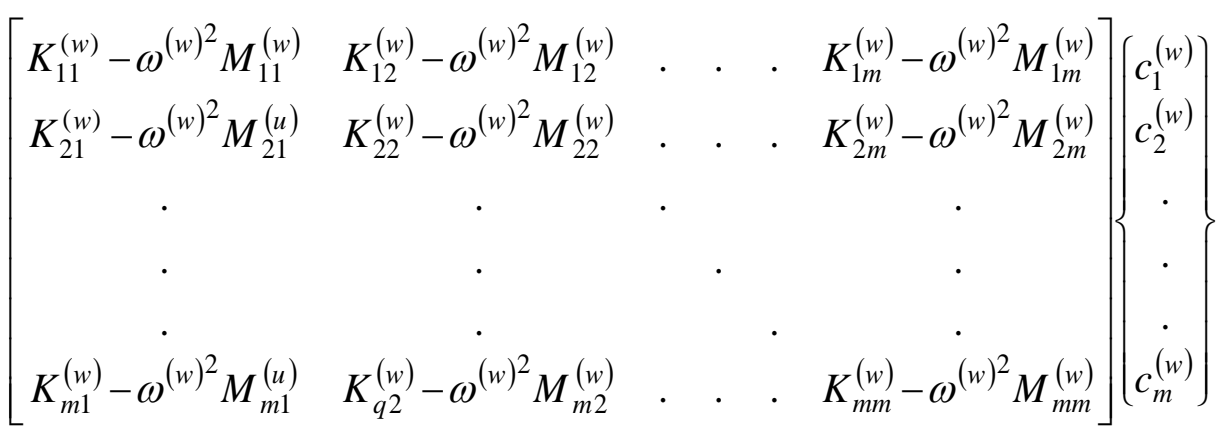

It should be noted that $c_{r}^{(u)}$ and $c_{r}^{(w)}$ are called the unknown Ritz coefficients for the respective longitudinal and transverse bending forms which refer to the eigenvectors in the mechanical domain. Since Eqs. (11) and (12) generate the set of m-eigenvalue algebraic equations, the m- 
eigenvectors for each eigenvalue can be stated as the generalized Ritz coefficients $c_{k r}^{(w)}$ and $c_{k r}^{(w)}$. At this point, the generalized space-dependent Ritz eigenfunctions can be formulated [17] as,

$$
\Psi_{r}(x)=\sum_{k=1}^{m} c_{k r}^{(w)} \Psi_{k}(x) \quad, \quad \Theta_{r}(x)=\sum_{k=1}^{m} c_{k r}^{(u)} \Theta_{k}(x) \quad r=1,2, \ldots, m
$$

The generalised Ritz mode shapes for Euler-Bernoulli bimorph beam can be normalised with respect to mass as,

$$
\begin{gathered}
\hat{\Psi}_{r}(x)=\frac{\Psi_{r}(x)}{\left(\int_{0}^{L} \hat{I}^{(A, k)} \Psi_{r}(x)^{2} \mathrm{~d} x+I_{\text {tip }}^{(A)} \Psi_{r}(L)^{2}+I_{\text {tip }}^{(C)}\left(\frac{\mathrm{d} \Psi_{r}}{\mathrm{~d} x}(L)\right)^{2}\right)^{1 / 2}} \quad \mathrm{r}=1,2, \ldots, \ldots, m \\
\hat{\Theta}_{r}(x)=\frac{\Theta_{r}(x)}{\left(\int_{0}^{L} \hat{I}^{(A, k)} \Theta_{r}(x)^{2} \mathrm{~d} x+I_{\text {tip }}^{(A)} \Theta_{r}(L)^{2}\right)^{1 / 2}} \quad \mathrm{r}=1,2, \ldots, m
\end{gathered}
$$

Corresponding to Eqs. (14) and (15), the normalised eigenfunction series forms can now be stated in terms of the generalised space- and time-dependent functions as,

$$
w_{r e l}(x, t)=\sum_{r=1}^{m} \hat{\Psi}_{r}(x) w_{r}(t) \quad, \quad u_{r e l}(x, t)=\sum_{r=1}^{m} \hat{\Theta}_{r}(x) u_{r}(t)
$$

Corresponding to Eqs. (3) and (4), the orthonormalisations can be proven by using Eq. (16) and applying the orthogonality property of the mechanical dynamic equations for EulerBernoulli bimorph beam [17] as,

$$
\begin{gathered}
\int_{0}^{L} \hat{I}^{(A, k)} \hat{\Psi}_{r}(x) \hat{\Psi}_{q}(x) \mathrm{d} x+I_{\text {tip }}^{(A)} \hat{\Psi}_{r}(L) \hat{\Psi}_{q}(L)+I_{\text {tip }}^{(C)} \frac{\mathrm{d} \hat{\Psi}_{r}(L)}{\mathrm{d} x} \frac{\mathrm{d} \hat{\Psi}_{q}(L)}{\mathrm{d} x}=\delta_{r q} \\
\int_{0}^{L} \hat{I}^{(A, k)} \hat{\Theta}_{r}(x) \hat{\Theta}_{q}(x) \mathrm{d} x+I_{\text {tip }}^{(A)} \hat{\Theta}_{r}(L) \hat{\Theta}_{q}(L)=\delta_{r q}, \int_{0}^{L} \hat{C}_{11}^{(D, k)} \frac{\mathrm{d} \hat{\Theta}_{r}(x)}{\mathrm{d} x} \frac{\mathrm{d} \hat{\Theta}_{q}(x)}{\mathrm{d} x} \mathrm{~d} x=\omega_{r}^{(u)^{2}} \delta_{r q} \\
\int_{0}^{L} \hat{C}_{11}^{(F, k)} \frac{\mathrm{d}^{2} \hat{\Psi}_{r}(x)}{\mathrm{d} x^{2}} \frac{\mathrm{d}^{2} \hat{\Psi}_{q}(x)}{\mathrm{d} x^{2}} \mathrm{~d} x=\omega_{r}^{(w)^{2}} \delta_{r q}
\end{gathered}
$$

where $\delta_{r q}$ is the Kronecker delta, defined as unity for $q=r$ and zero for $q \neq r$. The Rayleigh mechanical damping can be reduced in terms of orthonormalisation as,

$$
\begin{gathered}
C_{q r}^{(u)}=\alpha^{(u)} \delta_{q r}+\beta^{(u)} \omega_{r}^{(u)^{2}} \delta_{q r}=2 \zeta_{r}^{(u)} \omega_{r}^{(u)} \delta_{q r} \\
C_{q r}^{(w)}=\alpha^{(w)} \delta_{q r}+\beta^{(w)} \omega_{r}^{(w)^{2}} \delta_{q r}=2 \zeta_{r}^{(w)} \omega_{r}^{(w)} \delta_{q r}
\end{gathered}
$$


In this case, although the modal mechanical damping ratios can be determined mathematically, the chosen modal mechanical damping ratios $\zeta_{r}^{(u)}$ and $\zeta_{r}^{(w)}$ were obtained by experiment to give accurate results across the resonance frequency regions. Applying the orthonormalisations from Eqs. (17)-(19) into the electromechanical piezoelectric bimorph beam equation from Eq. (1) gives,

$$
\begin{gathered}
\ddot{u}_{r}(t)+2 \zeta_{r}^{(u)} \omega_{r}^{(u)} \dot{u}_{r}(t)+\omega_{r}^{(u)^{2}} u_{r}(t)+P_{r}^{(u)} v(t)=-Q_{r}^{(u)} \ddot{u}_{\text {base }}(t) \\
\ddot{w}_{r}(t)+2 \zeta_{r}^{(w)} \omega_{r}^{(w)} \dot{w}_{r}(t)+\omega_{r}^{(w)^{2}} w(t)+P_{r}^{(w)} v(t)=-Q_{r}^{(w)} \ddot{w}_{\text {base }}(t) \\
\hat{P}_{r}^{(u)} \dot{u}_{r}(t)+\hat{P}_{r}^{(w)} \dot{w}_{r}(t)+P_{D} \dot{v}(t)+R_{L} v(t)=0
\end{gathered}
$$

It is noted that because Eq. (22) has been normalised due to Eq. (16), the normalised parameters $P_{r}^{(u)}, P_{r}^{(w)}, \hat{P}_{r}^{(u)}, \hat{P}_{r}^{(w)}, P_{D}, Q_{r}^{(u)}$ and $Q_{r}^{(w)}$ can be reduced as,

$$
\begin{array}{r}
P_{r}^{(u)}=-\int_{0}^{L} \hat{R}_{31}^{(G, k)} \frac{\mathrm{d} \hat{\Theta}_{r}(x)}{\mathrm{d} x} \mathrm{~d} x, P_{r}^{(w)}=\int_{0}^{L} \hat{R}_{31}^{(H, k)} \frac{\mathrm{d}^{2} \hat{\Psi}_{r}(x)}{\mathrm{d} x^{2}} \mathrm{~d} x \\
\hat{P}_{r}^{(u)}=\sum_{r=0}^{m} P_{r}^{(u)}, \quad \hat{P}_{r}^{(w)}=\sum_{r=0}^{m} P_{r}^{(w)}, P_{D}=-\int_{0}^{L} \hat{S}_{33}^{(k)} \mathrm{d} x \\
Q_{r}^{(u)}=\int_{0}^{L} \hat{I}^{(A, k)} \hat{\Theta}_{r}(x) \mathrm{d} x+I_{\text {tip }}^{(A)} \hat{\Theta}_{r}(L), \quad Q_{r}^{(w)}=\int_{0}^{L} \hat{I}^{(A, k)} \hat{\Psi}_{r}(x) \mathrm{d} x+I_{\text {tip }}^{(A)} \hat{\Psi}_{r}(L)
\end{array}
$$

Equation (22) can be solved using Laplace transforms. In this case, the multi-mode electromechanical dynamic equations of the piezoelectric bimorph system can be written as,

$$
\begin{aligned}
& u_{r}(s)=-\frac{1}{Z(s)_{r}}\left[\left\{\left(s^{2}+2 \zeta_{r}^{(w)} \omega_{r}^{(w)} s+\omega_{r}^{(w)^{2}}\right)\left(s P_{D}+R_{L}\right)-\sum_{r=1}^{m} s P_{r}^{(w)^{2}}\right\} Q_{r}^{(u)}\left(s^{2} u_{\text {base }}(s)\right)\right. \\
& \left.+\sum_{r=1}^{m} s P_{r}^{(w)} P_{r}^{(u)} Q_{r}^{(w)}\left(s^{2} w_{\text {base }}(s)\right)\right] \\
& w_{r}(s)=-\frac{1}{Z(s)_{r}}\left[\left\{\left(s^{2}+2 \zeta_{r}^{(u)} \omega_{r}^{(u)} s+\omega_{r}^{(u)^{2}}\right)\left(s P_{D}+R_{L}\right)-\sum_{r=1}^{m} s P_{r}^{(u)^{2}}\right\} Q_{r}^{(w)}\left(s^{2} w_{\text {base }}(s)\right)\right. \\
& \left.+\sum_{r=1}^{m} s P_{r}^{(w)} P_{r}^{(u)} Q_{r}^{(u)}\left(s^{2} u_{\text {base }}(s)\right)\right] \\
& v(s)=\frac{1}{Z(s)_{r}}\left[\sum_{r=1}^{m}\left\{s P_{r}^{(w)} Q_{r}^{(w)}\left(s^{2}+2 \zeta_{r}^{(u)} \omega_{r}^{(u)} s+\omega_{r}^{(u)^{2}}\right)\left(s^{2} w_{\text {base }}(s)\right)\right\}\right. \\
& \left.+\sum_{r=1}^{m}\left\{s P_{r}^{(u)} Q_{r}^{(u)}\left(s^{2}+2 \zeta_{r}^{(w)} \omega_{r}^{(w)} s+\omega_{r}^{(w)^{2}}\right)\left(s^{2} u_{\text {base }}(s)\right)\right\}\right]
\end{aligned}
$$


The characteristic polynomial form from Eq. (22) can be formulated as,

$$
\begin{aligned}
Z(s)_{r} & =\left(s^{2}+2 \zeta_{r}^{(u)} \omega_{r}^{(u)} s+\omega_{r}^{(u)^{2}}\right)\left(s^{2}+2 \zeta_{r}^{(w)} \omega_{r}^{(w)} s+\omega_{r}^{(w)^{2}}\right)\left(s P_{D}+R_{L}\right) \\
& -\sum_{r=1}^{m} s P_{r}^{(w)^{2}}\left(s^{2}+2 \zeta_{r}^{(u)} \omega_{r}^{(u)} s+\omega_{r}^{(u)^{2}}\right)-\sum_{r=1}^{m} s P_{r}^{(u)^{2}}\left(s^{2}+2 \zeta_{r}^{(w)} \omega_{r}^{(w)} s+\omega_{r}^{(w)^{2}}\right)
\end{aligned}
$$

\subsection{Multi-Mode Electromechanical Frequency Response Functions}

Corresponding to Eqs. (23)-(25), the frequency response function (FRF) can be formulated by transforming $(s)$ with $(j \omega)$. After applying some simple algebra, the superposition of electromechanical frequency responses of the piezoelectric bimorph can be formulated as,

$$
\mathbf{G}(j \omega)=\mathbf{H}(j \omega) \mathbf{F}(j \omega)
$$

where the FRF in matrix form can be stated to give,

$$
\mathbf{H}(j \omega)=\left[\begin{array}{ll}
H_{11}(j \omega) & H_{12}(j \omega) \\
H_{21}(j \omega) & H_{22}(j \omega) \\
H_{31}(j \omega) & H_{32}(j \omega) \\
H_{41}(j \omega) & H_{42}(j \omega)
\end{array}\right]
$$

and the following output and input vector representations can be stated as,

$$
\mathbf{G}(j \omega)=\left[u_{r}(j \omega) w_{r}(j \omega) v(j \omega) I(j \omega)\right]^{T}, \quad \mathbf{F}(j \omega)=\left[-\omega^{2} u_{b a s e} e^{j \omega t}-\omega^{2} w_{b a s e} e^{j \omega t}\right]^{T}
$$

The first multi-mode FRF represents the generalised longitudinal displacement function with respect to input longitudinal acceleration. In the case, the FRF can be obtained from input base longitudinal excitation by ignoring the input transverse excitation as,

$$
\begin{aligned}
H_{11}(j \omega)=\left.\frac{u_{r}(j \omega)}{-\omega^{2} u_{\text {base }} e^{j \omega t}}\right|_{s=j \omega} \\
=-\frac{1}{\omega_{r}^{(u)^{2}}-\omega^{2}+j 2 \zeta_{r}^{(u)} \omega_{r}^{(u)} \omega}\left(\frac{\left(j \omega P_{D}+R_{L}\right) Q_{r}^{(u)}-\sum_{r=1}^{m} \frac{j \omega P_{r}^{(w)^{2}} Q_{r}^{(u)}}{\omega_{r}^{(w)^{2}}-\omega^{2}+j 2 \zeta_{r}^{(w)} \omega_{r}^{(w)} \omega}}{j \omega P_{D}+R_{L}-\sum_{r=1}^{m} \frac{j \omega P_{r}^{(w)^{2}}}{\omega_{r}^{(w)^{2}}-\omega^{2}+j 2 \zeta_{r}^{(w)} \omega_{r}^{(w)} \omega}-\sum_{r=1}^{m} \frac{j \omega P_{r}^{(u)^{2}}}{\omega_{r}^{(u)^{2}}-\omega^{2}+j 2 \zeta_{r}^{(u)} \omega_{r}^{(u)} \omega}}\right)
\end{aligned}
$$

Eq. (30) can be modified to obtain the FRF as a function of position of the piezoelectric element $(x)$ and frequency $(j \omega)$ by transforming it back into the normalised longitudinal Ritz eigenfunction form as,

$$
H_{11}(x, j \omega)=\left.\frac{u_{r e l}(x, j \omega)}{-\omega^{2} u_{b a s e} e^{j \omega t}}\right|_{s=j \omega}
$$




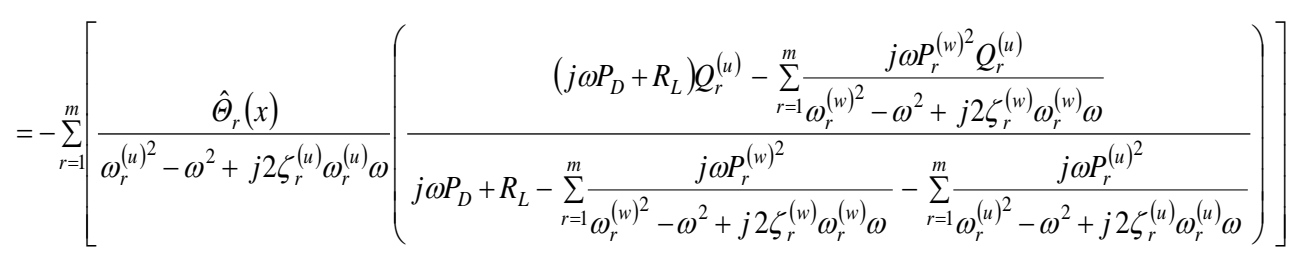

The FRF relating the input base transverse acceleration to the output longitudinal displacement can be obtained as,

$$
\begin{aligned}
& H_{12}(j \omega)=\left.\frac{u_{r}(j \omega)}{-\omega^{2} w_{\text {base }} e^{j \omega t}}\right|_{s=j \omega} \\
& =-\frac{1}{\omega_{r}^{(u)^{2}}-\omega^{2}+j 2 \zeta_{r}^{(u)} \omega_{r}^{(u)} \omega}\left(\frac{\sum_{r=1}^{m} \frac{j \omega P_{r}^{(u)} P_{r}^{(w)} Q_{r}^{(w)}}{\omega_{r}^{(\omega)}-\omega^{2}+j 2 \zeta_{r}^{(w)} \omega_{r}^{(w)} \omega}}{j \omega P_{D}+R_{L}-\sum_{r=1}^{m} \frac{j \omega P_{r}^{(w)^{2}}}{\omega_{r}^{(\omega)^{2}}-\omega^{2}+j 2 \zeta_{r}^{(w)} \omega_{r}^{(w)} \omega}-\sum_{r=1}^{m} \frac{j \omega P_{r}^{(u)^{2}}}{\omega_{r}^{(u)^{2}}-\omega^{2}+j 2 \zeta_{r}^{(u)} \omega_{r}^{(u)} \omega}}\right)
\end{aligned}
$$

Modifying Eq. (32) by transforming it back into the normalised Ritz longitudinal eigenfunction form gives,

$$
\begin{aligned}
& H_{12}(x, j \omega)=\left.\frac{u_{r e l}(x, j \omega)}{-\omega^{2} w_{b a s e} e^{j \omega t}}\right|_{s=j \omega} \\
& =-\sum_{r=1}^{m}\left[\frac{\hat{\Theta}_{r}(x)}{\omega_{r}^{(u)^{2}}-\omega^{2}+j 2 \zeta_{r}^{(u)} \omega_{r}^{(u)} \omega}\left(\frac{\sum_{r=1}^{m} \frac{j \omega P_{r}^{(u)} P_{r}^{(w)}-\omega^{2}+j 2 \zeta_{r}^{(w)} \omega_{r}^{(w)} \omega}{j \omega P_{D}+R_{L}-\sum_{r=1}^{m} \frac{j \omega P_{r}^{(w)^{2}}}{(w)^{2}}-\omega^{2}+j 2 \zeta_{r}^{(w)} \omega_{r}^{(w)} \omega}-\sum_{r=1}^{m} \frac{j \omega P_{r}^{(u)^{2}}}{\omega_{r}^{(u)^{2}}-\omega^{2}+j 2 \zeta_{r}^{(u)} \omega_{r}^{(u)} \omega}}{j}\right)\right]
\end{aligned}
$$

The multi-mode FRF represents the transverse displacement with respect to input longitudinal acceleration. If base-input transverse motion is ignored, the FRF of transverse motion related to the base input longitudinal excitation can be obtained as,

$$
\begin{aligned}
& H_{21}(j \omega)=\left.\frac{w_{r}(j \omega)}{-\omega^{2} u_{\text {base }} e^{j \omega t}}\right|_{s=j \omega} \\
& =-\frac{1}{\omega_{r}^{(\omega)^{2}}-\omega^{2}+j 2 \zeta_{r}^{(w)} \omega_{r}^{(w)} \omega}\left(\frac{\sum_{r=1}^{m} \frac{j \omega P_{r}^{(u)} P_{r}^{(w)} Q_{r}^{(u)}}{\omega_{r}^{(u)}-\omega^{2}+j 2 \zeta_{r}^{(u)} \omega_{r}^{(u)} \omega}}{j \omega P_{D}+R_{L}-\sum_{r=1}^{m} \frac{j \omega P_{r}^{(w)^{2}}}{\omega_{r}^{(w)^{2}}-\omega^{2}+j 2 \zeta_{r}^{(w)} \omega_{r}^{(\omega)} \omega}-\sum_{r=1}^{m} \frac{j \omega P_{r}^{(u)^{2}}}{\omega_{r}^{(u)^{2}}-\omega^{2}+j 2 \zeta_{r}^{(u)} \omega_{r}^{(u)} \omega}}\right)
\end{aligned}
$$

Corresponding to the normalised Ritz transverse eigenfunction form in Eq. (16a), Eq. (34) can be modified to obtain the FRF as a function of position of the piezoelectric element $(x)$ and frequency $(j \omega)$ as ,

$$
H_{21}(x, j \omega)=\left.\frac{w_{r e l}(x, j \omega)}{-\omega^{2} u_{b a s e} e^{j \omega t}}\right|_{s=j \omega}
$$




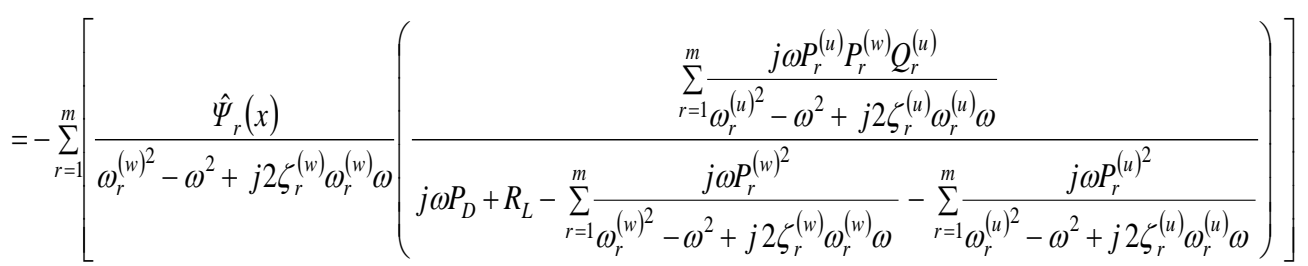

The multi-mode FRF of transverse displacement with respect to input base transverse acceleration can be obtained as,

$$
\begin{aligned}
& H_{22}(j \omega)=\left.\frac{w_{r}(j \omega)}{-\omega^{2} w_{\text {base }} e^{j \omega t}}\right|_{s=j \omega} \\
& =-\frac{1}{\omega_{r}^{(w)^{2}}-\omega^{2}+j 2 \zeta_{r}^{(w)} \omega_{r}^{(w)} \omega}\left(\frac{\left(j \omega P_{D}+R_{L}\right) Q_{r}^{(w)}-\sum_{r=1}^{m} \frac{j \omega P_{r}^{(u)^{2}} Q_{r}^{(w)}}{\omega_{r}^{(u)^{2}}-\omega^{2}+j 2 \zeta_{r}^{(u)} \omega_{r}^{(u)} \omega}}{j \omega P_{D}+R_{L}-\sum_{r=1}^{m} \frac{j \omega P_{r}^{(w)^{2}}}{\omega_{r}^{(\omega)^{2}}-\omega^{2}+j 2 \zeta_{r}^{(w)} \omega_{r}^{(w)} \omega}-\sum_{r=1}^{m} \frac{j \omega P_{r}^{(u)^{2}}}{\omega_{r}^{(u)^{2}}-\omega^{2}+j 2 \zeta_{r}^{(u)} \omega_{r}^{(u)} \omega}}\right)
\end{aligned}
$$

Eq. (36) can be modified by transforming it back into the normalised Ritz transverse eigenfunction to obtain the FRF as a function of position of the piezoelectric element $(x)$ and frequency $(j \omega)$ as,

$$
\begin{aligned}
& H_{22}(x, j \omega)=\left.\frac{w_{r e l}(x, j \omega)}{-\omega^{2} w_{\text {base }} e^{j \omega t}}\right|_{s=j \omega} \\
& =-\sum_{r=1}^{m}\left[\frac{\hat{\Psi}_{r}(x)}{\omega_{r}^{(w)^{2}}-\omega^{2}+j 2 \zeta_{r}^{(w)} \omega_{r}^{(w)} \omega}\left(\frac{\left.\left.\left(j \omega P_{D}+R_{L}\right) Q_{r}^{(w)}-\sum_{r=1}^{m} \frac{j \omega P_{r}^{(u)^{2}}-\omega^{2}+j 2 \zeta_{r}^{(w)} \omega_{r}^{(u)} \omega}{j \omega P_{D}+R_{L}-\sum_{r=1}^{m} \frac{j \omega P_{r}^{(w)^{2}}}{(w)^{2}}-\omega^{2}+j 2 \zeta_{r}^{(w)} \omega_{r}^{(w)} \omega}-\sum_{r=1}^{m} \frac{j \omega P_{r}^{(u)^{2}}}{\omega_{r}^{(u)^{2}}-\omega^{2}+j 2 \zeta_{r}^{(u)} \omega_{r}^{(u)} \omega}\right)\right]}{}\right)\right]
\end{aligned}
$$

The FRF relating the input base longitudinal acceleration to the output electric voltage can be obtained as,

$$
\begin{aligned}
& H_{31}(j \omega)=\left.\frac{v(j \omega)}{-\omega^{2} u_{\text {base }} e^{j \omega t}}\right|_{s=j \omega} \\
& =\frac{\sum_{r=1}^{m} \frac{j \omega P_{r}^{(u)} Q_{r}^{(u)}}{\omega_{r}^{(u)^{2}}-\omega^{2}+j 2 \zeta_{r}^{(u)} \omega_{r}^{(u)} \omega}}{j \omega P_{D}+R_{L}-\sum_{r=1}^{m} \frac{j \omega P_{r}^{(w)^{2}}}{\omega_{r}^{(w)^{2}}-\omega^{2}+j 2 \zeta_{r}^{(w)} \omega_{r}^{(w)} \omega}-\sum_{r=1}^{m} \frac{j \omega P_{r}^{(u)^{2}}}{\omega_{r}^{(u)^{2}}-\omega^{2}+j 2 \zeta_{r}^{(u)} \omega_{r}^{(u)} \omega}}
\end{aligned}
$$

The multi-mode FRF of the output electrical voltage with respect to the input base transverse excitation can be calculated, omitting the input base longitudinal excitation to give, 


$$
\begin{aligned}
& H_{32}(j \omega)=\left.\frac{v(j \omega)}{-\omega^{2} w_{\text {base }} e^{j \omega t}}\right|_{s=j \omega} \\
& =\frac{\sum_{r=1}^{m} \frac{j \omega P_{r}^{(w)} Q_{r}^{(w)}}{\omega_{r}^{(w)^{2}}-\omega^{2}+j 2 \zeta_{r}^{(w)} \omega_{r}^{(w)} \omega}}{j \omega P_{D}+R_{L}-\sum_{r=1}^{m} \frac{j \omega P_{r}^{(w)^{2}}}{\omega_{r}^{(w)^{2}}-\omega^{2}+j 2 \zeta_{r}^{(w)} \omega_{r}^{(w)} \omega}-\sum_{r=1}^{m} \frac{j \omega P_{r}^{(u)^{2}}}{\omega_{r}^{(u)^{2}}-\omega^{2}+j 2 \zeta_{r}^{(u)} \omega_{r}^{(u)} \omega}}
\end{aligned}
$$

The multi-mode FRF relating the input base longitudinal acceleration to the output electrical current can be obtained as,

$$
\begin{aligned}
& H_{41}(j \omega)=\left.\frac{I(j \omega)}{-\omega^{2} u_{\text {base }} e^{j \omega t}}\right|_{s=j \omega} \\
& =\frac{\frac{1}{R_{\text {load }}} \sum_{r=1}^{m} \frac{j \omega P_{r}^{(u)} Q_{r}^{(u)}}{\omega_{r}^{(u)^{2}}-\omega^{2}+j 2 \zeta_{r}^{(u)} \omega_{r}^{(u)} \omega}}{j \omega P_{D}+R_{L}-\sum_{r=1}^{m} \frac{j \omega P_{r}^{(w)^{2}}}{\omega_{r}^{(w)^{2}}-\omega^{2}+j 2 \zeta_{r}^{(w)} \omega_{r}^{(w)} \omega}-\sum_{r=1}^{m} \frac{j \omega P_{r}^{(u)^{2}}}{\omega_{r}^{(u)^{2}}-\omega^{2}+j 2 \zeta_{r}^{(u)} \omega_{r}^{(u)} \omega}}
\end{aligned}
$$

The FRF of the output electrical current with respect to the input base transverse excitation can be calculated, omitting the input base longitudinal excitation to give,

$$
\begin{aligned}
& H_{42}(j \omega)=\left.\frac{I(j \omega)}{-\omega^{2} w_{\text {base }} e^{j \omega t}}\right|_{s=j \omega} \\
& =\frac{\frac{1}{R_{\text {load }}} \sum_{r=1}^{m} \frac{j \omega P_{r}^{(w)} Q_{r}^{(w)}}{\omega_{r}^{(w)}-\omega^{2}+j 2 \zeta_{r}^{(w)} \omega_{r}^{(w)} \omega}}{j \omega P_{D}+R_{L}-\sum_{r=1}^{m} \frac{j \omega P_{r}^{(w)^{2}}}{\omega_{r}^{(w)^{2}}-\omega^{2}+j 2 \zeta_{r}^{(w)} \omega_{r}^{(w)} \omega}-\sum_{r=1}^{m} \frac{j \omega P_{r}^{(u)^{2}}}{\omega_{r}^{(u)^{2}}-\omega^{2}+j 2 \zeta_{r}^{(u)} \omega_{r}^{(u)} \omega}}
\end{aligned}
$$

The multi-mode FRF of power harvesting with respect to the input base longitudinal acceleration can be expressed as,

$$
\begin{aligned}
& \left.\frac{P(j \omega)}{\left(-\omega^{2} u_{\text {base }} e^{j \omega t}\right)^{2}}\right|_{s=j \omega} \\
& =\left[\frac{\frac{1}{\sqrt{R_{\text {load }}}} \sum_{r=1}^{m} \frac{j \omega P_{r}^{(u)} Q_{r}^{(u)}}{j \omega P_{r}^{(w)^{2}}-\omega^{2}+j 2 \zeta_{r}^{(u)} \omega_{r}^{(u)} \omega}}{j \omega P_{D}+R_{L}-\sum_{r=1}^{m} \frac{j \omega P_{r}^{(u)^{2}}}{\omega_{r}^{(w)^{2}}-\omega^{2}+j 2 \zeta_{r}^{(w)} \omega_{r}^{(w)} \omega}-\sum_{r=1}^{m} \frac{\omega_{r}^{(u)^{2}}-\omega^{2}+j 2 \zeta_{r}^{(u)} \omega_{r}^{(u)} \omega}{\omega^{2}}}\right]
\end{aligned}
$$

The multi-mode FRF of power harvesting with respect to the input transverse acceleration can be calculated as,

$$
\left.\frac{P(j \omega)}{\left(-\omega^{2} w_{b a s e} e^{j \omega t}\right)^{2}}\right|_{s=j \omega}
$$




$$
=\left[\frac{\frac{1}{\sqrt{R_{\text {load }}}} \sum_{r=1}^{m} \frac{j \omega P_{r}^{(w)} Q_{r}^{(w)}}{j \omega P_{r}^{(w)^{2}}-\omega^{2}+j 2 \zeta_{r}^{(w)} \omega_{r}^{(w)} \omega}-\sum_{r=1}^{m} \frac{j \omega P_{r}^{(u)^{2}}}{\omega_{r}^{(u)^{2}}-\omega^{2}+j 2 \zeta_{r}^{(u)} \omega_{r}^{(u)} \omega}}{j \omega P_{D}+R_{L}-\sum_{r=1}^{m} \frac{\omega_{r}^{(w)^{2}}-\omega^{2}+j 2 \zeta_{r}^{(w)} \omega_{r}^{(w)} \omega}{\omega^{2}}}\right]^{2}
$$

The optimal multi-mode FRF power harvesting related to the transverse acceleration can be calculated by differentiating with respect to load resistance and setting the differentiable power function to zero. It is noted that parameter $R_{L}$ represents per-unit load resistance $-1 / R_{\text {load }}$. Corresponding to Eq. (43), the optimal load resistance can be formulated as,

$$
R_{\text {load }}^{\text {opt }}=\frac{\sqrt{X(\omega)^{2}+Y(\omega)^{2}}}{X(\omega)^{2}+Y(\omega)^{2}}
$$

where

$$
\begin{array}{r}
X(\omega)=\omega P_{D}-\sum_{r=1}^{m} \frac{\omega P_{r}^{(w)^{2}}\left(\omega_{r}^{(w)^{2}}-\omega^{2}\right)}{\left(\omega_{r}^{(w)^{2}}-\omega^{2}\right)^{2}+\left(2 \zeta_{r}^{(w)} \omega_{r}^{(w)} \omega\right)^{2}}-\sum_{r=1}^{m} \frac{\omega P_{r}^{(u)^{2}}\left(\omega_{r}^{(u)^{2}}-\omega^{2}\right)}{\left(\omega_{r}^{(u)^{2}}-\omega^{2}\right)^{2}+\left(2 \zeta_{r}^{(u)} \omega_{r}^{(u)} \omega\right)^{2}} \\
Y(\omega)=\sum_{r=1}^{m} \frac{\omega P_{r}^{(w)^{2}}\left(2 \zeta_{r}^{(w)} \omega_{r}^{(w)} \omega\right)}{\left(\omega_{r}^{(w)}-\omega^{2}\right)^{2}+\left(2 \zeta_{r}^{(w)} \omega_{r}^{(w)} \omega\right)^{2}}-\sum_{r=1}^{m} \frac{\omega P_{r}^{(u)^{2}}\left(2 \zeta_{r}^{(u)} \omega_{r}^{(u)} \omega\right)}{\left(\omega_{r}^{(u)^{2}}-\omega^{2}\right)^{2}+\left(2 \zeta_{r}^{(u)} \omega_{r}^{(u)} \omega\right)^{2}}
\end{array}
$$

It should be noted that the optimal load resistance can be substituted back into Eq. (43) to give the optimal power harvesting.

Corresponding to Eqs. (30) and (32), Eq. (23) can be modified in terms of the generalised time-dependent longitudinal function as,

$$
\begin{gathered}
u_{r}(t)=H_{11}(j \omega) \ddot{u}_{\text {base }}+H_{12}(j \omega) \ddot{w}_{\text {base }} \\
u_{r}(t)=H_{11}(j \omega)\left(-\omega^{2} u_{\text {base }} e^{j \omega t}\right)+H_{12}(j \omega)\left(-\omega^{2} w_{\text {base }} e^{j \omega t}\right)
\end{gathered}
$$

Corresponding to Eq. (16b), modifying Eq. (45) in terms of any position along the piezoelectric beam gives the steady state relative longitudinal displacement under two input base excitations as,

$$
u_{r e l}(x, t)=H_{11}(x, j \omega)\left(-\omega^{2} u_{\text {base }} e^{j \omega t}\right)+H_{12}(x, j \omega)\left(-\omega^{2} w_{\text {base }} e^{j \omega t}\right)
$$

The multi-mode absolute longitudinal displacement can be formulated in terms of Eq. (46) as,

$$
u_{a b s}(x, t)=u_{\text {base }} e^{j \omega t}+H_{11}(x, j \omega)\left(-\omega^{2} u_{\text {base }} e^{j \omega t}\right)+H_{12}(x, j \omega)\left(-\omega^{2} w_{\text {base }} e^{j \omega t}\right)
$$


It should be noted that the absolute displacement field was formulated as $u_{a b s}(x, t)=u_{\text {base }}(t)+u_{r e l}(x, t)$. The generalised time dependent relative transverse displacement in Eq. (24) can be modified corresponding with Eqs. (34) and (36) as,

$$
\begin{gathered}
w_{r}(t)=H_{21}(j \omega) \ddot{u}_{\text {base }}+H_{22}(j \omega) \ddot{w}_{\text {base }} \\
w_{r}(t)=H_{21}(j \omega)\left(-\omega^{2} u_{\text {base }} e^{j \omega t}\right)+H_{22}(j \omega)\left(-\omega^{2} w_{\text {base }} e^{j \omega t}\right)
\end{gathered}
$$

Corresponding to Eq. (16a), Eq. (48) can be modified into the steady state relative transverse displacement in terms of any position on the piezoelectric beam as,

$$
w_{r e l}(x, t)=H_{21}(x, j \omega)\left(-\omega^{2} u_{\text {base }} e^{j \omega t}\right)+H_{22}(x, j \omega)\left(-\omega^{2} w_{b a s e} e^{j \omega t}\right)
$$

Corresponding to Eq. (49), the multi-mode absolute transverse displacement can be reduced as,

$$
w_{a b s}(x, t)=w_{b a s e} e^{j \omega t}+H_{21}(x, j \omega)\left(-\omega^{2} u_{b a s e} e^{j \omega t}\right)+H_{22}(x, j \omega)\left(-\omega^{2} w_{b a s e} e^{j \omega t}\right)
$$

It should be noted that Eq. (50) is formulated as $w_{a b s}(x, t)=w_{\text {base }}(t)+w_{r e l}(x, t)$. Eq. (25) can be modified into the generalised electrical potential response in terms of Eqs. (38) and (39) to give,

$$
\begin{gathered}
v(t)=H_{31}(j \omega) \ddot{u}_{\text {base }}+H_{32}(j \omega) \ddot{w}_{\text {base }} \\
v(t)=H_{31}(j \omega)\left(-\omega^{2} u_{\text {base }} e^{j \omega t}\right)+H_{32}(j \omega)\left(-\omega^{2} w_{\text {base }} e^{j \omega t}\right)
\end{gathered}
$$

Corresponding to Eqs. (40) and (41), the generalised electrical current response can be obtained by modifying Eq. (25) and then multiplying with $1 / R_{\text {load }}$ to give,

$$
\begin{gathered}
I(t)=H_{41}(j \omega) \ddot{u}_{\text {base }}+H_{42}(j \omega) \ddot{w}_{\text {base }} \\
I(t)=H_{41}(j \omega)\left(-\omega^{2} u_{\text {base }} e^{j \omega t}\right)+H_{42}(j \omega)\left(-\omega^{2} w_{\text {base }} e^{j \omega t}\right)
\end{gathered}
$$

Corresponding to Eqs. (47) and (50), Eqs. (31) and (37) can be modified to give the multimode FRF of the absolute displacements and velocities related to the base input longitudinal and transverse accelerations at any position along the bimorph respectively as,

$$
\begin{aligned}
& \hat{H}_{11}^{(d i s p)}(x, j \omega)=\frac{u_{\text {base }} e^{j \omega t}+u_{\text {rel }}(x, t)}{-\omega^{2} u_{\text {base }} e^{j \omega t}}=-\frac{1}{\omega^{2}}+H_{11}(x, j \omega) \\
& \hat{H}_{11}^{(v e l)}(x, j \omega)=\frac{\frac{\mathrm{d}}{\mathrm{d} t}\left[u_{\text {base }} e^{j \omega t}+u_{\text {rel }}(x, t)\right]}{-\omega^{2} u_{\text {base }} e^{j \omega t}}=\frac{1}{j \omega}+j \omega H_{11}(x, j \omega) \\
& \hat{H}_{22}^{(d i s p)}(x, j \omega)=\frac{w_{\text {base }} e^{j \omega t}+w_{r e l}(x, t)}{-\omega^{2} w_{\text {base }} e^{j \omega t}}=-\frac{1}{\omega^{2}}+H_{22}(x, j \omega)
\end{aligned}
$$




$$
\hat{H}_{22}^{(v e l)}(x, j \omega)=\frac{\frac{\mathrm{d}}{\mathrm{d} t}\left[w_{\text {base }} e^{j \omega t}+w_{\text {rel }}(x, t)\right]}{-\omega^{2} w_{\text {base }} e^{j \omega t}}=\frac{1}{j \omega}+j \omega H_{22}(x, j \omega)
$$

It should be noted that Eqs. (47), (50) and (55) are applicable for analysing the absolute dynamic responses when comparing the results using the Laser Doppler Vibrometer (LDV) because the measured signal output from the Vibrometer can be transferred into a digital signal FFT Analyzer to display the results. The results obtained from measurements can be the time dependent absolute displacement, velocity, acceleration, frequency spectrum and frequency response function located at any position along the piezoelectric bimorph.

\section{Experimental Validation}

In this section, the application of the electromechanical dynamic responses of the piezoelectric bimorph beam under two input base longitudinal and transverse excitations are considered by simulation and experiment. The results obtained from the analytical method can be validated by comparison with the experimental study. The chosen properties of the piezoelectric bimorph with centre brass shim were based on the PZT PSI-5A4E from Piezo Systems, INC. including tip mass as given in Table 1. Two types of experiments were conducted. First, the cantilevered piezoelectric bimorph beam with the tip mass was clamped at the base structure to provide only input base transverse excitation. Second, the rigid protractor base structure was used with incremental bimorph angle of $22.5^{\circ}$ in order to vary the direction of input acceleration and to investigate the resulting bimorph electromechanical dynamic responses. The B \& K impedance head type 8001, connected to the B \& K Charge Amplifier Type 2635, was used to measure the input acceleration from the B \& K exciter type 4809. Since the generating vibration signal amplitude needs to be regulated, the exciter was connected to the B \& K Power Amplifier Type 2706. Moreover, the wave function generator, connected to the Power Amplifier, was used to create specific harmonic input excitation. The vibration of the tip mass located at the end of the bimorph was measured using a laser digital vibrometer Polytec PDV 100 by attaching a small piece of reflector tape onto the tip mass to measure the absolute dynamic displacement, velocity and frequency responses. All signal measurements from the charge amplifier, piezoelectric bimorph and vibrometer were connected to the B \& K FFT Pulse Analyzer 3560B. The analyzer displayed the measurement results using the Pulse software with subsequent analysis conducted using the MATLAB software. The complete experimental setup is shown in Fig. 2. 
The FRF obtained from the analytical analysis can be used to model the piezoelectric bimorph with either series or parallel connection and a purely resistive load. In this case, the parallel connection was chosen for the example theoretical results. The FRF can be used to model and analyse the frequency, displacement, velocity, voltage, current and power harvesting based on the chosen varying load resistances. The FRF was investigated with changing resistances of $560 \Omega, 5.6 \mathrm{k} \Omega, 20 \mathrm{k} \Omega, 30 \mathrm{k} \Omega, 51 \mathrm{k} \Omega, 60 \mathrm{k} \Omega, 79 \mathrm{k} \Omega, 150 \mathrm{k} \Omega, 200 \mathrm{k} \Omega$ and $602 \mathrm{k} \Omega$. The mechanical damping ratios were determined experimentally with a load resistance of $560 \Omega$ approaching short circuit. The damping ratios for the transverse and longitudinal forms around the fundamental resonant frequency were found to be $\zeta_{1}^{w}=0.0139$ and $\zeta_{1}^{u}=0.030$. In this case, the absolute dynamic displacement (considered as the total dynamic displacement) can also be obtained due to the input base motion and the relative dynamic displacement. The input acceleration for the analysis here was $306 \mathrm{mg}$ (1 $\mathrm{g}=$ gravitational acceleration $9.81 \mathrm{~m} / \mathrm{s}^{2}$ ).

As can be seen from Figs. 3 and 5, the tip absolute dynamic displacement and velocity FRFs at the first resonance frequency shifts along the frequency axis as the load resistance changes. When the load resistance tended toward short circuit at the frequency of $76.1 \mathrm{~Hz}$, the amplitudes (absolute displacement and velocity) tended to increase with reducing resistance. Opposite behaviour was found as the resistance approached the open circuit response at the frequency of $79.6 \mathrm{~Hz}$, with increased response with increasing resistance. This indicated that the effect of the lowest and highest load resistances of $58 \Omega$ and $602 \mathrm{k} \Omega$ on the bimorph tended to reduce the sensitivity of the electrical form around the resonance frequency region due to the dominant mechanical behaviour of the bimorph showing the highest amplitudes. Moreover, as shown in Fig. 4, tip absolute transverse displacement from the short to open circuit resonance frequencies of 76.1 and $79.6 \mathrm{~Hz}$, respectively seemed to have similar trend pattern and different amplitude values with tip absolute velocity as shown in Fig. 6. The results shown from the tip displacement and velocity responses indicated close agreement between the experimental and analytical models with changing load resistance.

As can be seen from Fig. 7, the first mode FRF of electrical voltage also gave close agreement between theoretical and experimental results under varying resistance. The trend of electrical voltage for short circuit conditions tended to give the lowest amplitude whereas the open circuit load gave the highest amplitude. In this case, the increase of load resistance from the short to open circuits resulted in an increasing voltage amplitude followed by an 
increasing shift in the resonance frequency. The off-resonance frequency regions also gave increased voltage response with increasing resistance. Furthermore, Fig. 8 also shows very good agreement between the measured and simulated short and open circuit resonance amplitudes versus load resistance. The maximum electrical voltage amplitudes with the short and open circuit resonances can be reached with increasing load resistance. However, the maximum level of the open circuit resonance amplitude indicated a higher value compared with the short circuit when the short and open circuit amplitudes passed over the transitional point of $60 \mathrm{k} \Omega$.

As shown in Fig. 9, there was a slight increase of electrical current amplitude with decreasing load resistance followed by decreasing resonance frequencies. In this case, the electrical current frequency response with varying load resistance shows the electromechanical attenuation behaviour. The trend of electrical current shows a monotonic pattern opposite to that of the electric voltage response shown in Fig. 7. The analytical and experimental results gave very good agreement under varying load resistances as shown in Fig. 9. The short circuit frequency response seemed to give the highest amplitude at load resistance of $560 \Omega$ and resulted in the lowest resonance around $76.1 \mathrm{~Hz}$ compared to other load resistances. The load resistance of $5.6 \mathrm{k} \Omega$ still indicated short circuit behaviour although the amplitude obtained was a bit lower than the load resistance of $560 \Omega$, while the resonance frequency still indicated the same value. The open circuit load resistance of $602 \mathrm{k} \Omega$ gave the lowest current amplitude with the higher resonance frequency compared with other load resistances. Moreover, the maximum amplitudes of electrical current for both short and open circuit resonance frequencies under varying load resistance was achieved with decreasing load resistance as shown in Fig. 10. The level of maximum current amplitudes from short and open circuit resonances was shown to be different. In this point, the short circuit resonance amplitude below the transitional point of $60 \mathrm{k} \Omega$ gave higher current value compared with the open circuit resonance amplitude.

The comparison of electrical power harvesting frequency response of the bimorph with varying load resistance is presented in Fig. 11 with close agreement between the theoretical and experimental results. It should be noted that the power harvesting resonance frequency response also shifts as the load resistance changes, showing a combination of trends from the voltage and current responses as expected. The FRF of voltage approaching short circuit conditions gave the lowest voltage amplitude whereas the FRF of electrical current seemed to 
give the highest current amplitude with the same resonance frequency. This indicates that the power harvesting under short and open circuit conditions will not be optimal because although the tip absolute displacement or velocity were at maximum value, the electrical current and voltage amplitude results appeared to have opposite trends to each other. The result is that the short or open circuit resistance regions provide the lowest amplitude of power.

Two maximum points from the power harvesting response curves in Fig. 11 involved load resistances of $20 \mathrm{k} \Omega$ and $200 \mathrm{k} \Omega$, respectively, with the local minimum curve having resistance of $60 \mathrm{k} \Omega$. The local maximum points showed higher transverse displacement and velocity amplitudes and also gave higher power harvesting results compared with the local minimum point obtained from the $60 \mathrm{k} \Omega$ resistance. The optimal power harvesting amplitude occurred with the load resistance of $60 \mathrm{k} \Omega$ over the resonance frequency region. This gave the lowest level of tip absolute dynamic displacement or velocity amplitude and was located at the intermediate curves from the dynamic displacement, velocity, electrical current and voltage. The short and open circuit resonance frequency power results are shown in Fig. 12 under various load resistances showing that they reach the maximum power level with different load resistances as expected.

Another important aspect of predicting power harvesting can be shown by setting the base protractor structure onto the piezoelectric bimorph with different angles (polar form) to simulate the effect of having input excitation with different orientation. The application of this case can be found in most industrial environments where the input base vibration source will give multidirectional inputs onto the piezoelectric beam structure. It should be noted that the majority of mechanical strain in the bimorph interlayer results from the transverse input base motion.

The input base acceleration of the exciter for the polar tests was kept at a constant value of $3 \mathrm{~m} / \mathrm{s}^{2}$ for every angle. The resulting polar form results are shown in Fig. 13 for different frequencies and resistances. In this case, the angles of the base protractor structure were setup from $0^{\circ}-180^{\circ}$ with the incremental angles of $22.5^{\circ}$. The polar power results show the symmetrical response for the angles from $0^{\circ}-90^{\circ}$ and $90^{\circ}-180^{\circ}$ as expected with very close agreement between the analytical and experimental results. The maximum power was measured at the angle of $90^{\circ}$ due to the dominant transverse motion of the bimorph as expected from the model. The lowest power can be found at the angles of $0^{\circ}$ and $180^{\circ}$ due to the dominant longitudinal input base motion at this condition. The polar power harvesting results shown in Figs. 13a and $13 \mathrm{~b}$ for the load resistances of $20 \mathrm{~K} \Omega$ and $150 \mathrm{k} \Omega$ tended to 
overlap each other. This situation can also be found in the results with the angle of $90^{\circ}$ shown in Fig. 11 where the two amplitudes of power from the load resistances of $20 \mathrm{k} \Omega$ and $150 \mathrm{k} \Omega$ coincide for the frequencies of $77.71 \mathrm{~Hz}$ and $72.67 \mathrm{~Hz}$. The comparisons between the analytical and experimental results still showed reasonable agreement. The polar power harvesting should be seen to be a result of the combination of both the transverse and longitudinal system response. Similar behaviour of power was also found from the protractor angles of $180^{\circ}-270^{\circ}$ and $270^{\circ}-360^{\circ}$.

\section{Conclusions}

This paper has presented the comparison between analytical and experimental studies of a cantilevered piezoelectric bimorph beam with one and two input base transverse and longitudinal excitations. The resulting non-homogeneous electromechanical matrix differential equations were obtained using the weak form from Hamiltonian's principle. The formulations can be used to model either series or parallel connections of the piezoelectric bimorph beam. In this case, we chose to present only the parallel connection as shown in the theoretical and experimental results. The solution forms were based on the normalised Ritz eigenfunctions and were manipulated using Laplace transforms to obtain the FRFs. The resulting analytical model provides multi-input dynamic excitation and multi-output electromechanical dynamic responses including time series-dependent electromechanical responses. As the formulations were given in terms of the multi-mode FRFs, the single mode, which is the main concern for power harvesting, can be easily obtained from the multi-mode. The results obtained from the single mode FRFs showed the changes in resonance frequency based on the load resistance changes. The comparisons between the analytical and experimental results were achieved with good agreement. Moreover, the experimental findings show that the maximum tip absolute displacement tended to give the lowest and highest electrical voltage for resistance values approaching the short and open circuit conditions respectively. Conversely, the maximum tip absolute displacement tended to give the highest and lowest electrical current approaching the short and open circuits conditions, respectively. This shows that the maximum dynamic displacement does not necessarily result in the highest current or voltage. The short and open circuit conditions were found to give the lowest amount of power. This indicates that the power harvesting can be maximised without maximising displacement, current or voltage. This situation was shown clearly for the load resistance value around $60 \mathrm{k} \Omega$ where the displacement at this load resistance showed the 
lowest value but also gave convenient amplitudes for the electrical current, voltage and power across a broad frequency range. Finally, the polar power harvesting electromechanical dynamic response of the bimorph with the tip mass under two input base transverse and longitudinal accelerations also showed close agreement between the analytical and experimental results. The maximum power was achieved when the bimorph beam was under input transverse excitation at the angle of $90^{\circ}$ as expected. Although the combination of input excitations (transverse and longitudinal) to the base bimorph can be shown with the interval angle of $0^{\circ}<\theta<90^{\circ}$ or $90^{\circ}<\theta<180^{\circ}$, the generated power still resulted primarily from the transverse bending in the bimorph element where the input base transverse excitation played the major role of exciting the bimorph. 


\section{Appendix A. Determining mass moment of inertias of the piezoelectric bimorph and tip} mass

Coefficient mass moment of inertias from Eq. (1) can be simply formulated in terms of geometry and material characteristics of the piezoelectric bimorph. In terms of Fig. 1, the zero-th mass moment of inertia of the piezoelectric bimorph was given as,

$$
I^{(A, k)}=2 h_{p} \rho^{(A, 1)}+b h_{s} \rho^{(A, 2)}
$$

The densities $\rho^{(A, 1)}$ and $\rho^{(A, 3)}$ represent the material located within the upper and lower piezoelectric layers respectively and $\rho^{(A, 3)}$ represents the material of the middle layer of the brass shim. Furthermore, the second mass moment of inertia represents the rotary inertia of the bimorph which is formulated as,

$$
I^{(C, k)}=\left(\frac{2}{3}\left(h_{p}+\frac{h_{s}}{2}\right)^{3}-\frac{h_{s}{ }^{3}}{12}\right) \rho^{(C, 1)}+\frac{h_{s}^{3}}{4} \rho^{(C, 2)},
$$

where the densities $\rho^{(C, 1)}$ and $\rho^{(C, 3)}$ denote the same material located within the upper and lower piezoelectric layers respectively. It should be noted that $\rho^{(A, 1)}=\rho^{(A, 3)}=\rho^{(C, 1)}=\rho^{(C, 3)}$ and $\rho^{(A, 2)}=\rho^{(C, 2)}$. The zeroth mass moment of inertia of the proof mass based on Fig. 1 can also be formulated as,

$$
I_{\text {tip }}^{(A)}=\left(h_{\text {tip }} l_{\text {tip }}-\left(2 h_{p}+h_{s}\right) l_{b}\right) s_{\text {tip }} \rho_{\text {tip }}^{(A)}
$$

The second mass moment of inertia which is called the rotary inertia at the centre of gravity of the proof mass, can be written,

$$
I_{\text {tip }}^{(C)}=\left\{\left(\frac{\left(l_{\text {tip }}^{2}+h_{\text {tip }}^{2}\right)}{12}+\bar{x}_{1}^{2}-\frac{\left(\left(2 h_{p}+h_{s}\right)^{2}+l_{b}^{2}\right)}{12}-\bar{x}_{2}{ }^{2}\right)\left(l_{\text {tip }} h_{\text {tip }}-\left(2 h_{p}+h_{s}\right) l_{b}\right)\right\} s_{\text {tip }} \rho_{\text {tip }}^{(C)}
$$

where $\bar{x}_{1}=x_{g}-l_{\text {tip }} / 2, \bar{x}_{2}=x_{g}-l_{b} / 2$ and $x_{g}$ is the centre of gravity of the tip mass. 
Appendix B. Determining stiffness coefficients for the piezoelectric bimorph interlayer

The extensional stiffness coefficient of the piezoelectric bimorph can be stated as,

$$
C_{11}^{(D, k)}=2 h_{p} \bar{Q}_{11}^{(D, 1)}+h_{s} \bar{Q}_{11}^{(D, 2)}
$$

The transverse stiffness coefficient can be formulated as,

$$
C_{11}^{(F, k)}=\left(\frac{2}{3}\left(h_{p}+\frac{h_{s}}{2}\right)^{3}-\frac{h_{s}^{3}}{12}\right) \bar{Q}_{11}^{F, 1}+\frac{h_{s}^{3}}{4} \bar{Q}_{11}^{F, 2}
$$

where $\bar{Q}_{11}$ represents the plane stress-based elastic stiffness of the bimorph beam. 


\section{Appendix C. Determining forward and backward piezoelectric coupling coefficients and internal capacitance of the piezoelectric bimorph}

The piezoelectric coupling $\hat{R}_{31}$ in terms of series and parallel electrical connections will be discussed in detail and new techniques for formulating the piezoelectric coupling will also be given. The electric field of the piezoelectric bimorph depends on the positive and negative terminals located on the lower and upper surfaces of the piezoelectric element, respectively. Each connection (the series and parallel connections) can be arranged into two types of poled configurations i.e. X-poled and Y-Poled which depends on the direction of polarities and strain effect between the piezoelectric benders (upper element and lower element). As considered previously, the piezoelectric bimorph is not only assumed to undergo pure transverse bending but it also undergoes additional deformation i.e. longitudinal extension, which is reflected in the strain fields of the Euler-Bernoulli's beam expression. This will affect the polarisation of the piezoelectric bimorph which depends on strain at the lower and upper layers due to input mechanical vibration and also the chosen type of connections. The piezoelectric bimorph is assumed to have symmetrical geometry with the same material in the upper and lower layers and a brass centre shim. At this point, when the piezoelectric element was initially undeformed, the polarisation direction, for example, was in the z-axis (perpendicular to the bimorph length) giving the initial polarized state. Subsequently, when tensile stress acts perpendicular to the z-axis on the element, the polarisation will behave in the opposite direction to the z-axis. Conversely, when the piezoelectric element is under compressive stress perpendicular to the z-axis, the polarisation will be in the same direction as the z-axis. This indicates that the change of stress from tensile to compressive or vice versa in the piezoelectric element will result in a reversal of the direction of polarisation [18]. This situation is known as the direct piezoelectric effect where the polarisation is proportional to the stress field and the stress field is also proportional to the strain field which can be stated in terms of Einstein's summation convention as $P_{i}=d_{i j} \sigma_{j}$. At this point, the polarisation of the piezoelectric bimorph undergoes X-poling and Y-poling in one type of connection because the piezoelectric bimorph was considered mathematically under two input-base excitations using coupling superposition of the elastic-polarity field. When the piezoelectric bimorph beam is arranged for parallel connection as shown in Fig. C.1, two poling vectors cross in the piezoelectric material (upper and lower layers), which is X-poled (opposite polarisations between upper and lower layers) due to the longitudinal extensional term and Ypoled (same polarisations between upper and lower layers) due to the transverse bending 
term. On the other hand, the series connection with the same material can also have two poling effects given by the $\mathrm{Y}$-poling due to the longitudinal extension term and X-poling due to the transverse bending term. In this case, the cantilever piezoelectric bimorph with two input excitations modelled both electrical connection types. It is noted that $R_{31}^{(G, k)}$ represents the backward and forward piezoelectric couplings for the longitudinal extension term and $R_{31}^{(H, k)}$ represents the backward and forward piezoelectric couplings for the transverse bending term.

Case I. Series electrical connection.

a) Piezoelectric coupling for X-poling due to transverse bending can be formulated as,

$$
R_{31}^{(H, k)}=-\frac{e_{31}^{(H, 1)}}{2 h_{p}}\left(\frac{h_{p}{ }^{2}}{2}+\frac{h_{p} h_{s}}{2}\right)-\frac{e_{31}^{(H, 3)}}{2 h_{p}}\left(\frac{h_{p}{ }^{2}}{2}+\frac{h_{p} h_{s}}{2}\right)=-\frac{e_{31}}{h_{p}}\left(\frac{h_{p}{ }^{2}}{2}+\frac{h_{p} h_{s}}{2}\right)
$$

b) Piezoelectric coupling for Y-poling due to longitudinal extension can also be stated as,

$$
R_{31}^{(G, k)}=\frac{h_{p} e_{31}^{(G, 1)}}{2 h_{p}}+\frac{h_{p} e_{31}^{(G, 3)}}{2 h_{p}}=e_{31}
$$

The capacitance of the piezoelectric element was calculated as,

$$
S_{33}^{(k)}=\frac{h_{p} \varsigma_{33}^{(1)}}{4 h_{p}{ }^{2}}+\frac{h_{p} \varsigma_{33}^{(3)}}{4 h_{p}{ }^{2}}=\frac{\varsigma_{33}^{\varepsilon}}{2 h_{p}}
$$

It should be note that the upper and lower layers of the piezoelectric bimorph will have the same material and geometrical structure, thus the permittivity of the piezoelectric element will be $\varsigma_{33}^{(1)}=\varsigma_{33}^{(3)}=\varsigma_{33}^{\varepsilon}$. It should be noted that $\varsigma_{33}^{\varepsilon}$ is the permittivity at constant strain that can be formulated as $\varsigma_{33}^{\varepsilon}=\varsigma_{33}^{\sigma}-e_{31} d_{31}$ or $\varsigma_{33}^{\varepsilon}=\varsigma_{33}^{\sigma}-d_{31}^{2} \bar{Q}_{11}^{E}$ where $\bar{Q}_{11}^{E}=1 / s_{11}^{E}, \varsigma_{33}^{\sigma}$ is the permittivity at constant stress and $s_{11}^{E}$ is the elastic compliance at constant electric field.

Case II. Parallel electrical connection

a) Piezoelectric coupling for X-poling due to longitudinal extension can be formulated as,

$$
R_{31}^{(G, k)}=e_{31}^{(G, 1)}+e_{31}^{(G, 3)}=2 e_{31}
$$

b) Piezoelectric coupling for Y-poling due to transverse bending can be formulated as,

$$
R_{31}^{(H, k)}=-\frac{e_{31}^{(H, 1)}}{h_{p}}\left(\frac{h_{p}{ }^{2}}{2}+\frac{h_{p} h_{s}}{2}\right)-\frac{e_{31}^{(H, 3)}}{h_{p}}\left(\frac{h_{p}{ }^{2}}{2}+\frac{h_{p} h_{s}}{2}\right)=-\frac{2 e_{31}}{h_{p}}\left(\frac{h_{p}{ }^{2}}{2}+\frac{h_{p} h_{s}}{2}\right)
$$


The capacitance of the piezoelectric element for parallel connection was given by,

$$
S_{33}^{(k)}=\frac{h_{p} \varsigma_{33}^{(1)}}{h_{p}{ }^{2}}+\frac{h_{p} \varsigma_{33}^{(3)}}{h_{p}{ }^{2}}=\frac{2 \varsigma_{33}^{\varepsilon}}{h_{p}}
$$




\section{Appendix D. Determining the space-dependent eigenfunction forms of the Euler- Bernoulli piezoelectric bimorph beam with tip mass}

As mentioned previously, the space-dependent eigenfunctions can be used to develop the solution forms of the dynamic equations (Eq. (7)). To obtain the solution forms for Eqs. (3), (4) and (6), mechanical dynamic equations of transverse bending and longitudinal extension for the cantilevered piezoelectric bimorph beam with tip mass must be established independently to obtain the mode shapes or space-dependent eigenfunctions. These mode shapes are solution forms of the eigenfunction series to be used in Eq. (7). Firstly, the transverse bending dynamic equation for the cantilevered piezoelectric bimorph beam can be formulated as,

$$
\frac{\mathrm{d}^{4} \Psi_{r}(x)}{\mathrm{d} x^{4}}-\mu^{4} \Psi_{r}(x)=0
$$

If rotary inertia from the piezoelectric bimorph is ignored, then $\mu^{4}=\hat{I}^{(A, k)} \omega^{2} / \hat{C}_{11}^{(F, k)}$ will give four roots,

$$
\lambda_{1,2}= \pm \mu \quad, \quad \lambda_{3,4}= \pm j \mu
$$

The boundary condition of the transverse bending equation can be formulated with the tip mass and rotary moment of inertia from the tip mass as,

$$
\begin{gathered}
\hat{C}_{11}^{(F, k)} \frac{\mathrm{d}^{2} \Psi_{r}}{\mathrm{~d} x^{2}}(L)-I_{\text {tip }}^{(C)} \omega^{2} \frac{\mathrm{d} \Psi_{r}}{\mathrm{~d} x}(L)=0 \\
\hat{C}_{11}^{(F, k)} \frac{\mathrm{d}^{3} \Psi_{r}}{\mathrm{~d} x^{3}}(L)+I_{t i p}^{(A)} \omega^{2} \Psi_{r}(L)=0 \\
\Psi_{r}(0)=0 \quad, \quad \frac{\mathrm{d} \Psi_{r}}{\mathrm{~d} x}(0)=0
\end{gathered}
$$

The characteristic equation can be obtained after manipulation to give,

$$
\left[\begin{array}{ll}
A_{11} & A_{12} \\
A_{21} & A_{22}
\end{array}\right]\left\{\begin{array}{l}
c_{1} \\
c_{4}
\end{array}\right\}=0
$$

where,

$$
\begin{aligned}
& A_{11}=-(\cos (\mu L)+\cosh (\mu L))+\frac{I_{\text {tip }}^{(C)} \mu^{3}}{\hat{I}^{(A, k)}}(\sin (\mu L)+\sinh (\mu L)) \\
& A_{12}=(\sin (\mu L)+\sinh (\mu L))+\frac{I_{\text {tip }}^{(C)} \mu^{3}}{\hat{I}^{(A, k)}}(\cos (\mu L)-\cosh (\mu L)) \\
& A_{21}=(\sin (\mu L)-\sinh (\mu L))+\frac{I_{\text {tip }}^{(A)} \mu}{\hat{I}^{(A, k)}}(\cos (\mu L)-\cosh (\mu L))
\end{aligned}
$$




$$
A_{22}=(\cos (\mu L)+\cosh (\mu L))-\frac{I_{\text {tip }}^{(A)} \mu}{\hat{I}^{(A, k)}}(\sin (\mu L)-\sinh (\mu L))
$$

The frequency equation and eigenvalues can be calculated by analysing the determinant from Eq. (D3) to give,

$$
\begin{aligned}
& (1+\cos (\mu L) \cosh (\mu L))-\frac{I_{\text {tip }}^{(C)} \mu^{3}}{\hat{I}^{(A, k)}}(\cos \mu L \sinh \mu L+\sin \mu L \cosh \mu L) \\
& +\frac{I_{\text {tip }}^{(C)} \mu}{\hat{I}^{(A, k)}}(\cos \mu L \sinh \mu L-\sin \mu L \cosh \mu L)+\frac{I_{\text {tip }}^{(C)^{2}} \mu^{4}}{\hat{I}^{(A, k)^{2}}}(1-\cos \mu L \cosh \mu L)=0
\end{aligned}
$$

After applying boundary conditions and some algebraic calculations, the mode shape can now be formulated as,

$$
\Psi_{r}(x)=c_{1 r}\left(\cos (\mu x)-\cosh (\mu x)+\frac{\mathrm{A}_{21}}{\mathrm{~A}_{22}}(\sin (\mu x)-\sinh (\mu x))\right)
$$

Secondly, the dynamic equation of longitudinal motion for the cantilevered piezoelectric bimorph beam can be written as,

$$
\frac{\mathrm{d}^{2} \Theta(x)}{\mathrm{d} x^{2}}+\gamma^{2} \Theta(x)=0
$$

Eq. (D7) can be modified into a characteristic equation and two roots from the characteristic equation can be obtained as,

$$
\lambda_{1,2}= \pm j \gamma
$$

where, $\quad \gamma^{2}=\frac{\omega^{2} \hat{I}^{(A, k)}}{\hat{C}_{11}^{(D, k)}}$

The boundary condition can be formulated as,

$$
\hat{C}_{11}^{(D, k)} \frac{\mathrm{d} \Theta}{\mathrm{d} x}(L)-I_{\text {tip }}^{(A)} \omega^{2} \Theta(L)=0 \quad, \quad \Theta(0)=0
$$

The frequency equation and eigenvalues can be calculated by applying boundary conditions to give,

$$
\tan \gamma L=\frac{\hat{C}_{11}^{(D, k)} \gamma}{I_{t i p}^{(A)} \omega^{2}}
$$

After applying boundary conditions and some algebraic calculations, the mode shape can now be formulated as,

$$
\Theta_{r}(x)=b_{1 r} \sin \gamma x
$$




\section{References}

[1] I. Howard, A review of rolling element bearing vibration : detection, diagnosis and prognosis, Defence Science and Technology Organisation, Australian Department of Defence, DSTO-RR-0013 (1994).

[2] C.G. Knight, Wireless networks : Implications for aircraft loads monitoring, Defence Science and Technology Organisation, Australian Department of Defence, DSTO-TN0759 (2007).

[3] S. Roundy, P. Wright, J. Rabaey, A study of low level vibrations as a power source for wireless sensor nodes, Computer Communications 26 (2003) 1131-1144.

[4] N.E. duToit, B.L. Wardle, S-G. Kim, Design considerations for MEMS-scale piezoelectric mechanical vibration energy harvesters, Journal of Integrated Ferroelectrics 71 (2005) 121-160.

[5] N.E. duToit, B.L. Wardle, Performance of micro fabricated piezoelectric vibration energy harvesters, Integrated Ferroelectrics 83 (2006) 13-32.

[6] M. Kim, M. Hoegen, J. Dugundji, B.L. Wardle, Modeling and experimental verification of proof mass effects on vibration energy harvester performance, Smart Materials and Structures 19 (2010) 045023.

[7] A. Erturk, D.J. Inman, An experimentally validated bimorph cantilevered model for Piezoelectric energy harvesting from base excitations, Smart Materials and Structures 18 (2009) 1-18.

[8] F. Goldschmidtboeing, P. Woias, Characterization of different beam shapes for piezoelectric energy harvesting, Journal of Micromechanics and Microengineering, 18 (2008) 104013.

[9] M. Renaud, P. Fiorini, C.V. Hoof, Optimization of a piezoelectric unimorph for shock and impact energy harvesting, Smart Materials and Structures 16 (2007) 1125-1135.

[10] Y.C. Shu, I.C. Lien, Analysis of power output for piezoelectric energy harvesting systems, Smart Materials and Structures 15 (2006) 1499-1512.

[11] A. Mathers, K.S. Moon, J. Yi, A Vibration-based PMN-PT energy harvester, IEEE Sensor Journal, 9 (7) (2009) 731-739.

[12] R.D. Mindlin, Equations of high frequency vibrations of thermopiezoelectric crystal plates, International Journal of Solid and Structures 10 (1974) 625-637.

[13] H.F. Tiersten, Linear Piezoelectric Plate Vibrations, Plenum Press, 1969.

[14] A. Fernandes, J. Pouget, Analytical and numerical approaches to piezoelectric bimorph, International Journal of Solid and Structures, 40 (2003) 4331-4352 
[15] J.H. Huang, Y.C. Shiah, B.J. Lee, Electromechanical responses of a long piezoelectric tube subjected to dynamic loading, Journal of Physics D: Applied Physic 41 (2008) 025404

[16] M.F. Lumentut, I.M. Howard, The analysis of a piezoelectric bimorph beam with two input base motions for power harvesting, IOP Conf. Series: Material Science and Engineering 10 (2010) 012169.

[17] M.F. Lumentut, I.M. Howard, Analytical Modeling of self-powered electromechanical piezoelectric bimorph beams with multidirectional excitation, International Journal of Smart and Nano Materials (2011) in press.

[18] M. F. Lumentut, I.M. Howard, The experimental validation of an electromechanical dynamic model of a piezoelectric bimorph beam for prediction of power generation, Proceedings of the $6^{\text {th }}$ Australasian Congress on Applied Mechanics (2010) 908-917.

[19] J.F. Nye, Physical properties of crystals: their representation by tensors and matrices, Clarendon Press, Oxford, England, 1985. 
Table 1 Characteristic properties of the piezoelectric bimorph system.

\begin{tabular}{llllcc}
\hline Material properties & Piezoelectric & Brass & Geometry properties & Piezoelectric Brass \\
\hline Young's modulus, $\bar{Q}_{11}(\mathrm{GPa})$ & 66 & 105 & Length,$L(\mathrm{~mm})$ & 30.1 & 30.1 \\
Density, $\rho\left(\mathrm{kg} / \mathrm{m}^{3}\right)$ & 7800 & 9000 & Thickness, $h(\mathrm{~mm})$ & $0.19($ each $)$ & 0.13 \\
Piezoelectric constant, $d_{31}(\mathrm{pm} / \mathrm{V})$ & -190 & - & Width, $b(\mathrm{~mm})$ & 6.4 & 6.4 \\
Permittivity, $\varsigma_{33}^{\sigma}(\mathrm{F} / \mathrm{m})$ & $1800 \varsigma_{\mathrm{o}}$ & - & First coefficient $I_{\text {tip }}^{(A)}(\mathrm{kg})^{\dagger}$ & 0.0022 \\
permittivity of free space, $\varsigma_{\mathrm{o}}(\mathrm{pF} / \mathrm{m})$ & 8.854 & - & Third coefficient $I_{\text {tip }}^{(C)}\left(\mathrm{kg} \mathrm{m}^{2}\right)^{\dagger}$ & $7.3743 \times 10^{-9}$ \\
\hline
\end{tabular}

† Calculated according to the geometry and material properties of tip mass and the rotary inertia at centre of gravity of tip mass

coincident with the end of the bimorph length as shown in Fig. 1 where $l_{\text {tip }}=8.1 \mathrm{~mm}, h_{\text {tip }}=5.7 \mathrm{~mm}, l_{0}=5 \mathrm{~mm}$ and $s_{\text {tip }}=6.4 \mathrm{~mm}$ (width).

First and third coefficients refer to zeroth and second mass moment of inertias respectively

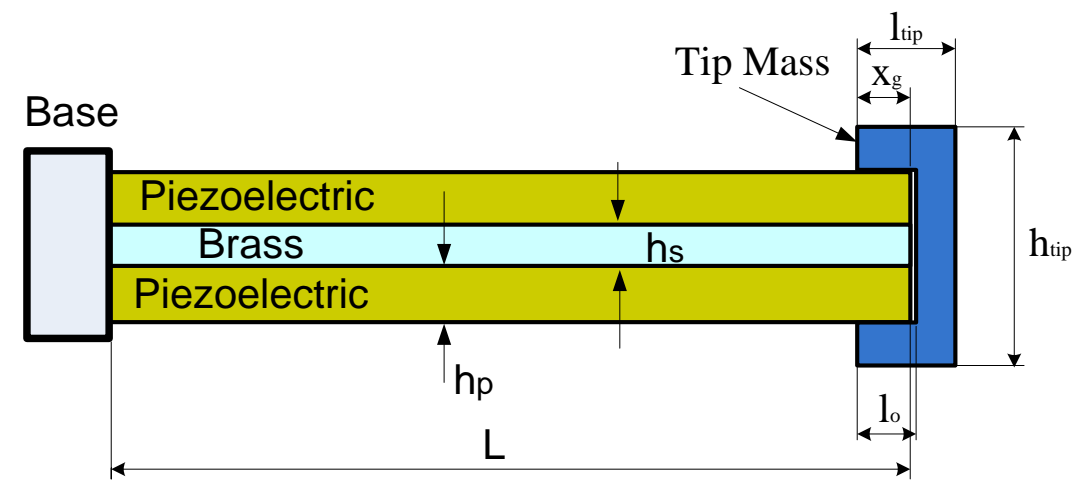

Fig. 1. Piezoelectric Bimorph Beam with a Tip Mass

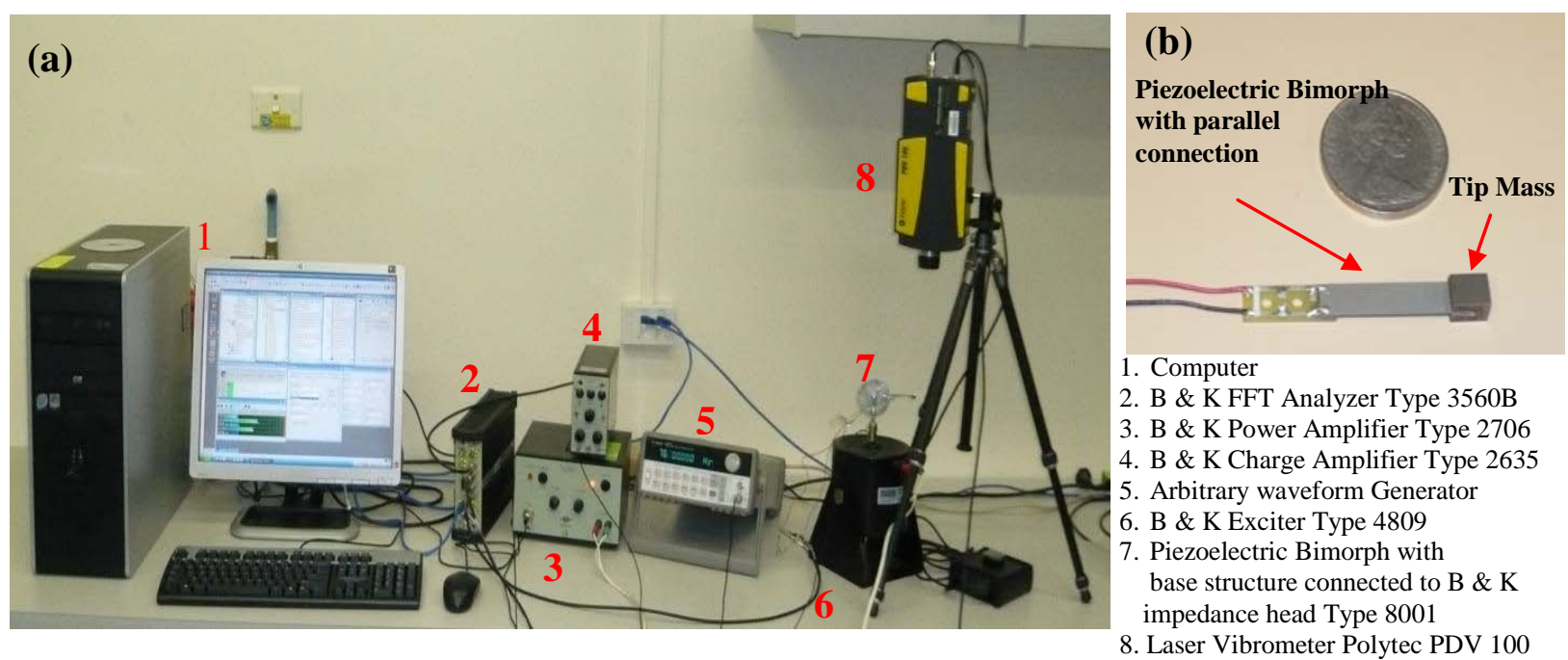

Fig. 2. (a) Experimental Setup and (b) Piezoelectric bimorph beam with tip mass under parallel connection 


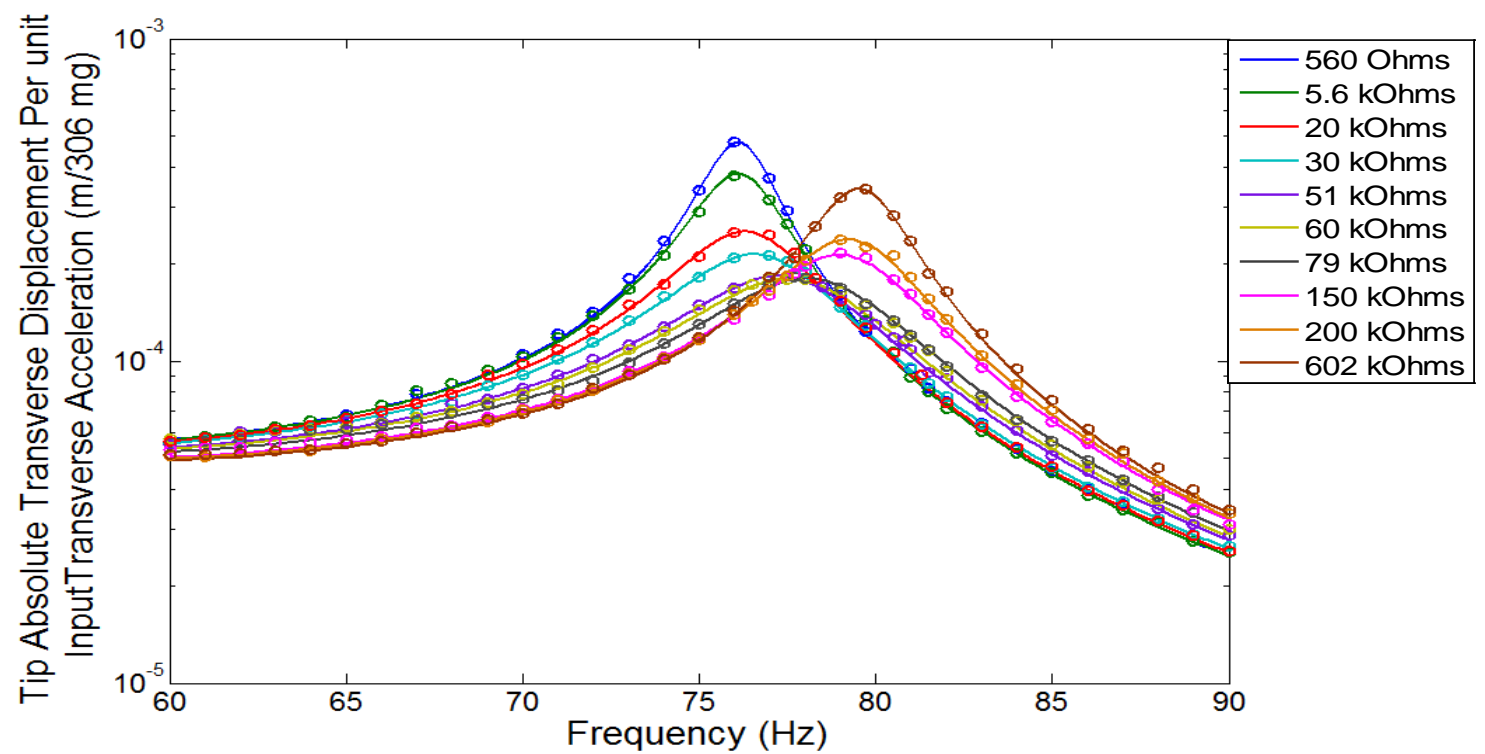

Fig. 3. FRFs of tip absolute Dynamic Displacement Analytical results (Solid line) and Experiment (Round dot)

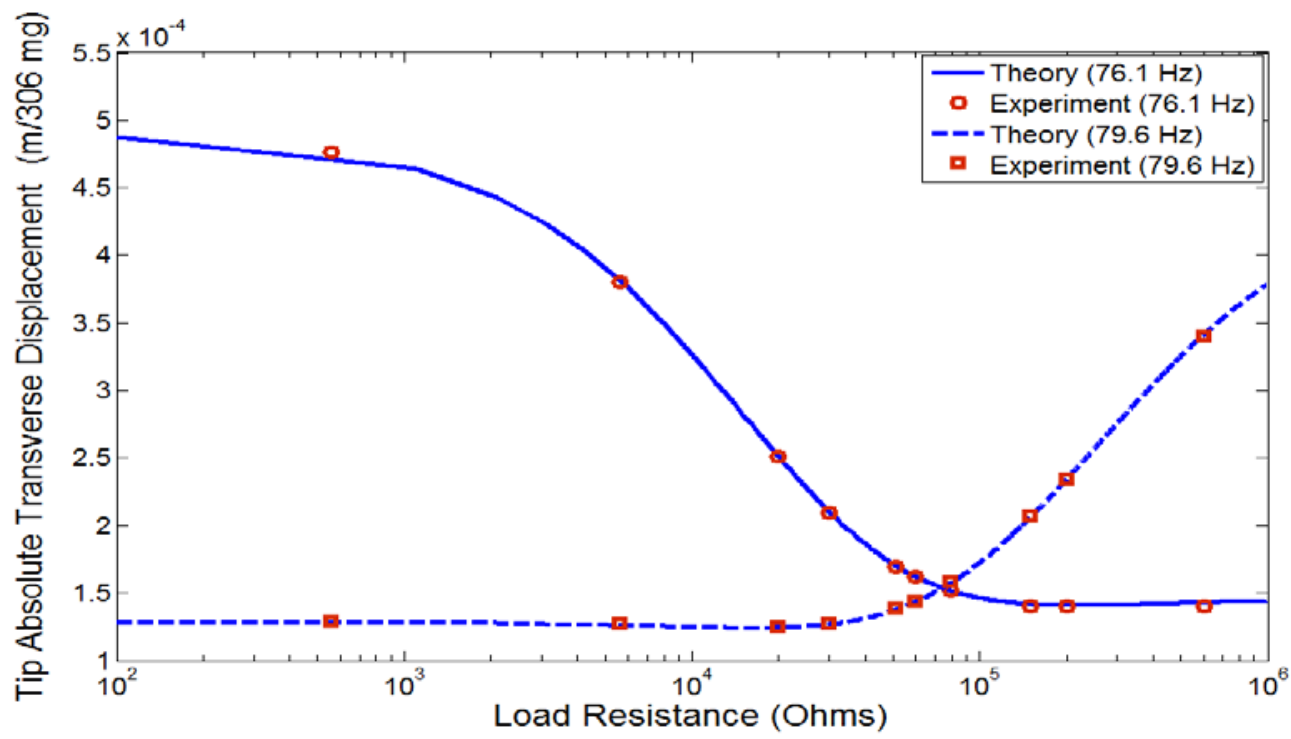

Fig. 4. Tip absolute Dynamic Displacement versus Load Resistance under the Short circuit Resonance Frequency of $76.1 \mathrm{~Hz}$ and Open Circuit Resonance Frequency of $79.6 \mathrm{~Hz}$

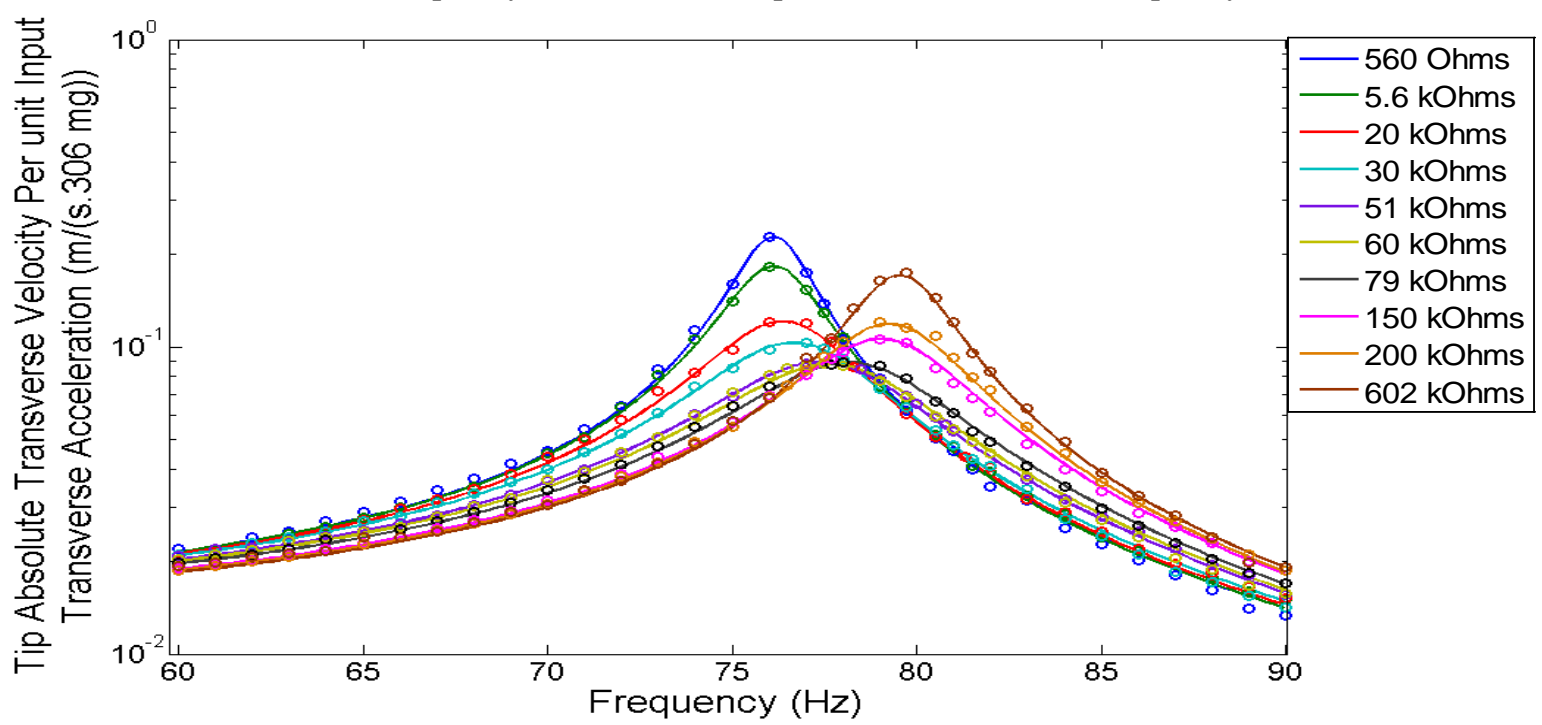

Fig. 5. FRFs of tip absolute Dynamic Velocity with Analytical results (Solid line) and Experiment (Round dot) 


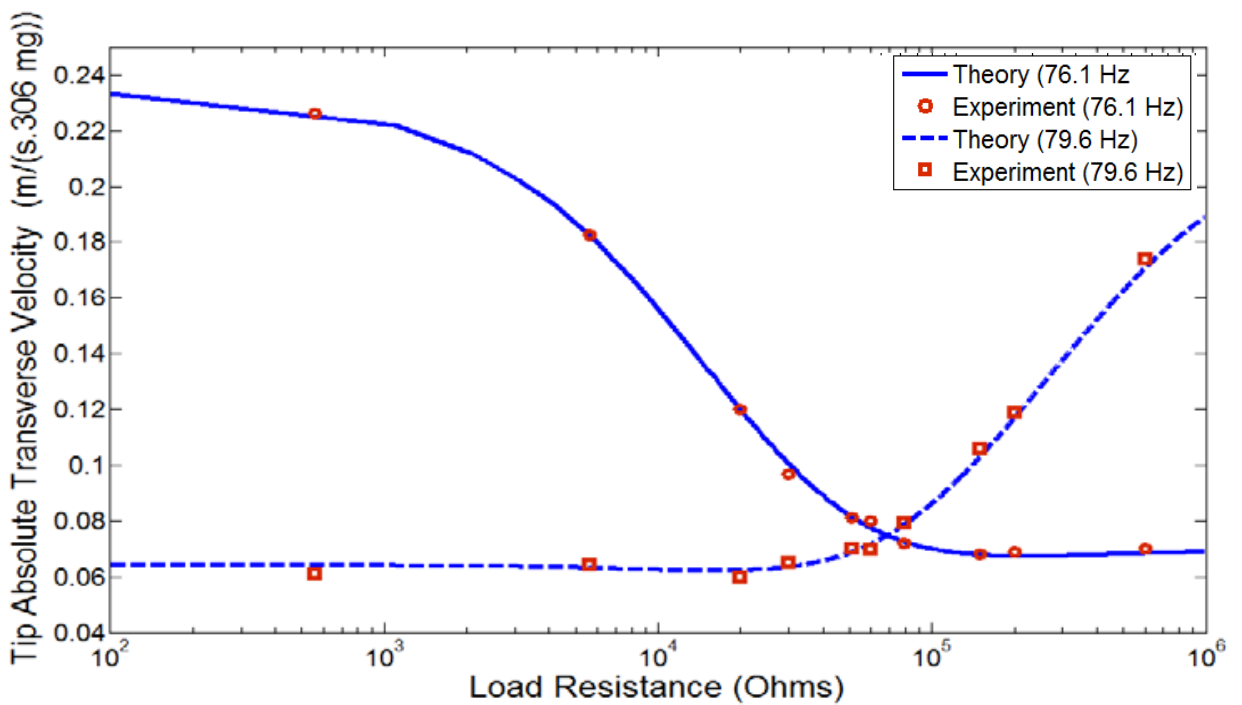

Fig. 6. Tip absolute Dynamic Velocity versus Load Resistance under the Short circuit Resonance Frequency of $76.1 \mathrm{~Hz}$ and Open Circuit Resonance Frequency of $79.6 \mathrm{~Hz}$

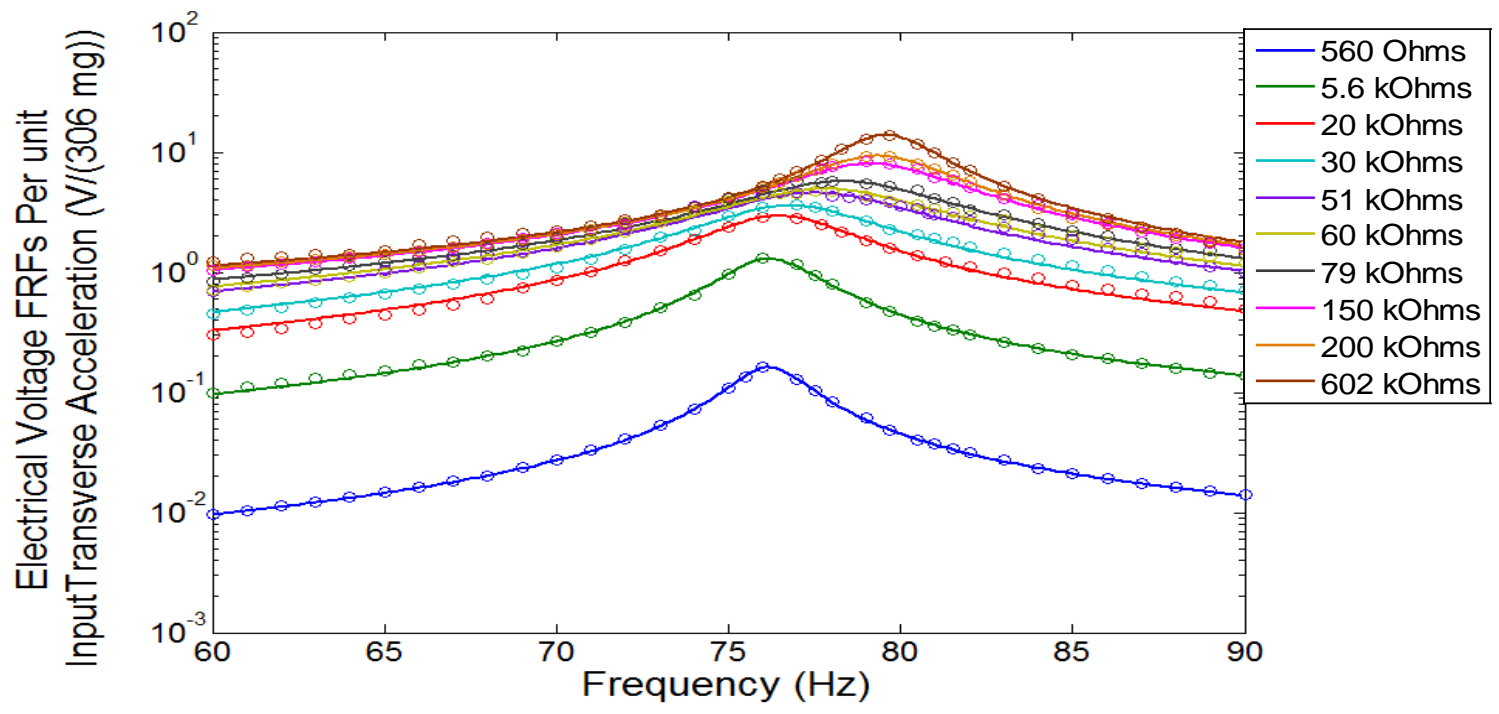

Fig.7. FRFs of Electrical Voltage with the Analytical results (Solid line) and Experiment (Round dot)

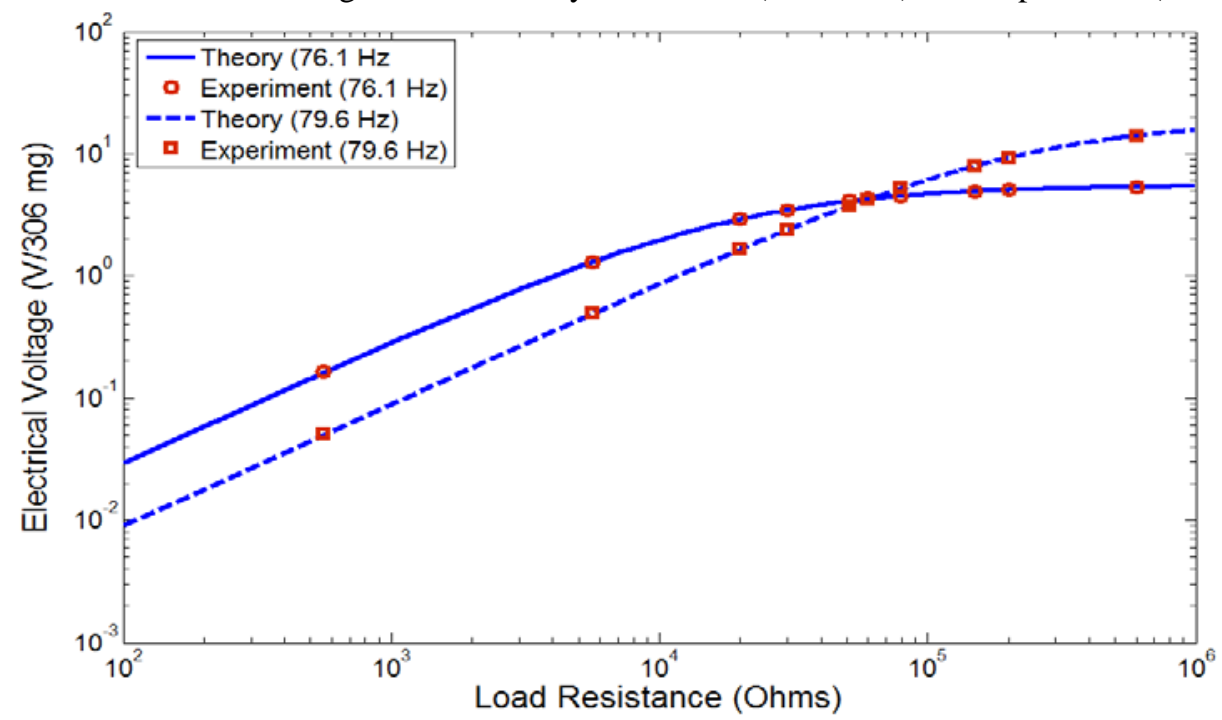

Fig. 8. Electrical Voltage versus Load Resistance under the Short circuit Resonance Frequency of $76.1 \mathrm{~Hz}$ and Open Circuit Resonance Frequency of $79.6 \mathrm{~Hz}$ 


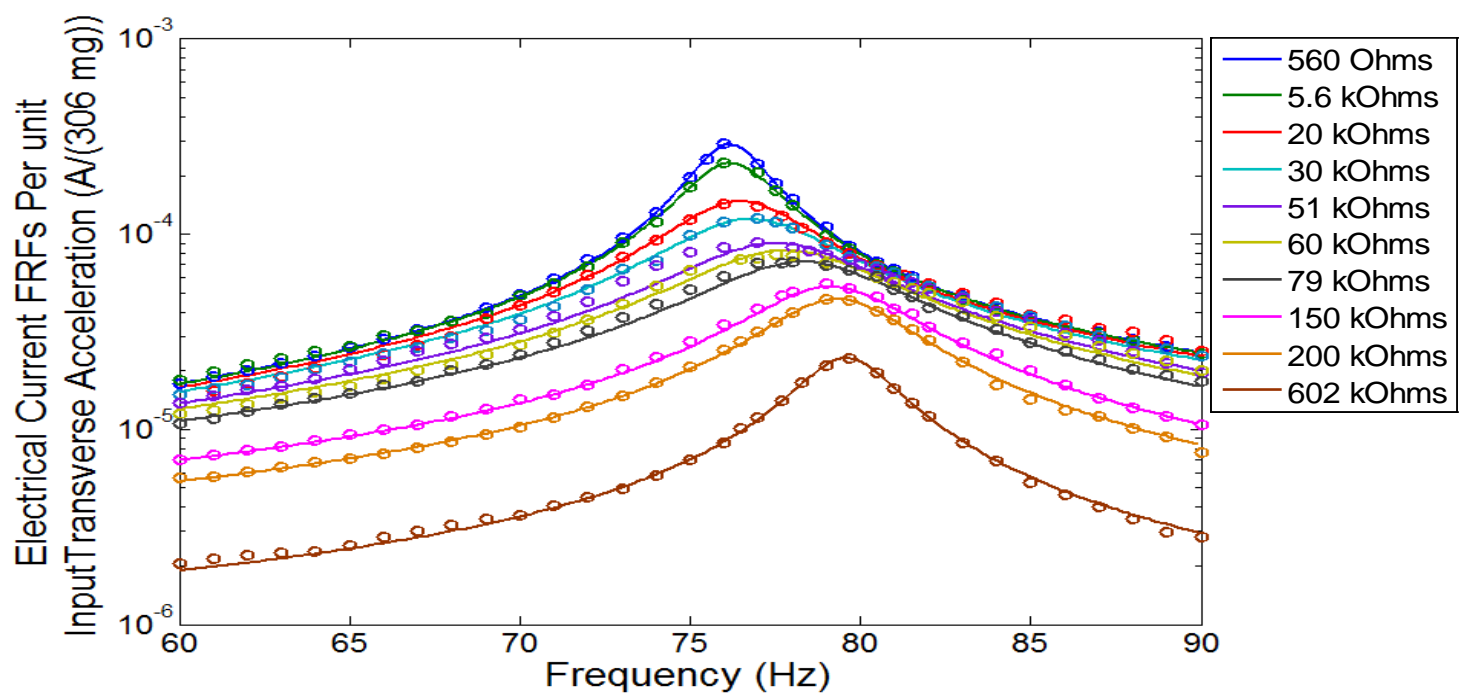

Fig. 9. FRFs of Electrical Current with Analytical results (Solid line) and Experiment (Round dot)

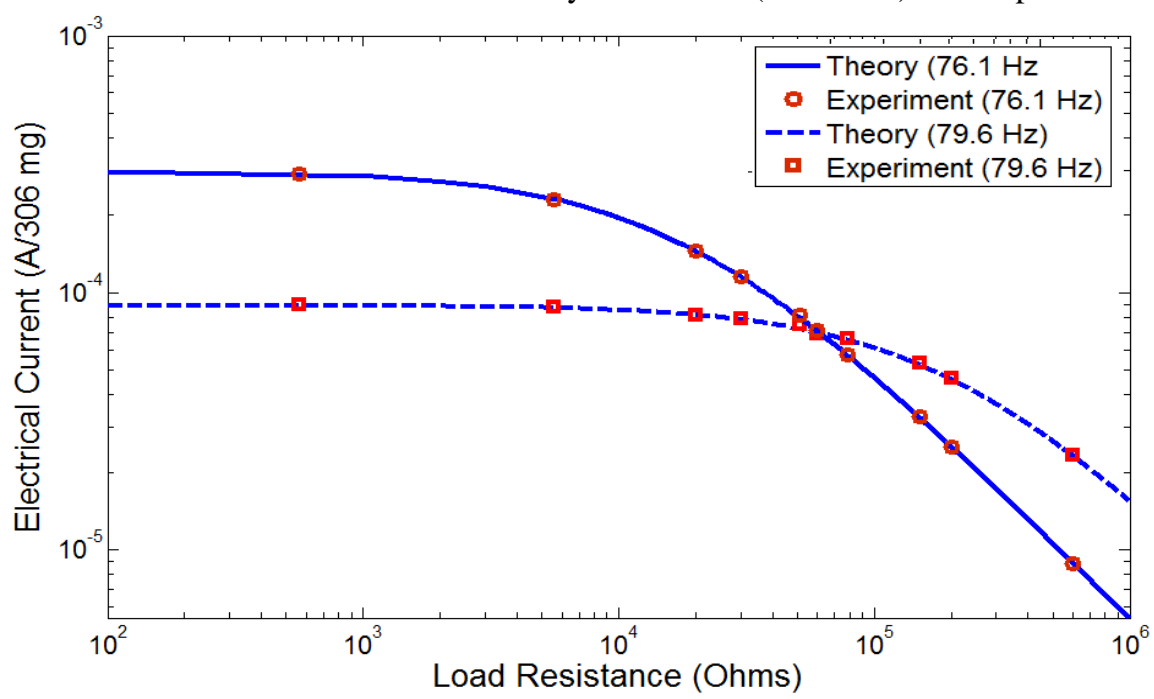

Fig. 10. Electrical Current versus Load Resistance under the Short circuit Resonance Frequency of $76.1 \mathrm{~Hz}$ and Open Circuit Resonance Frequency of 79.6 Hz

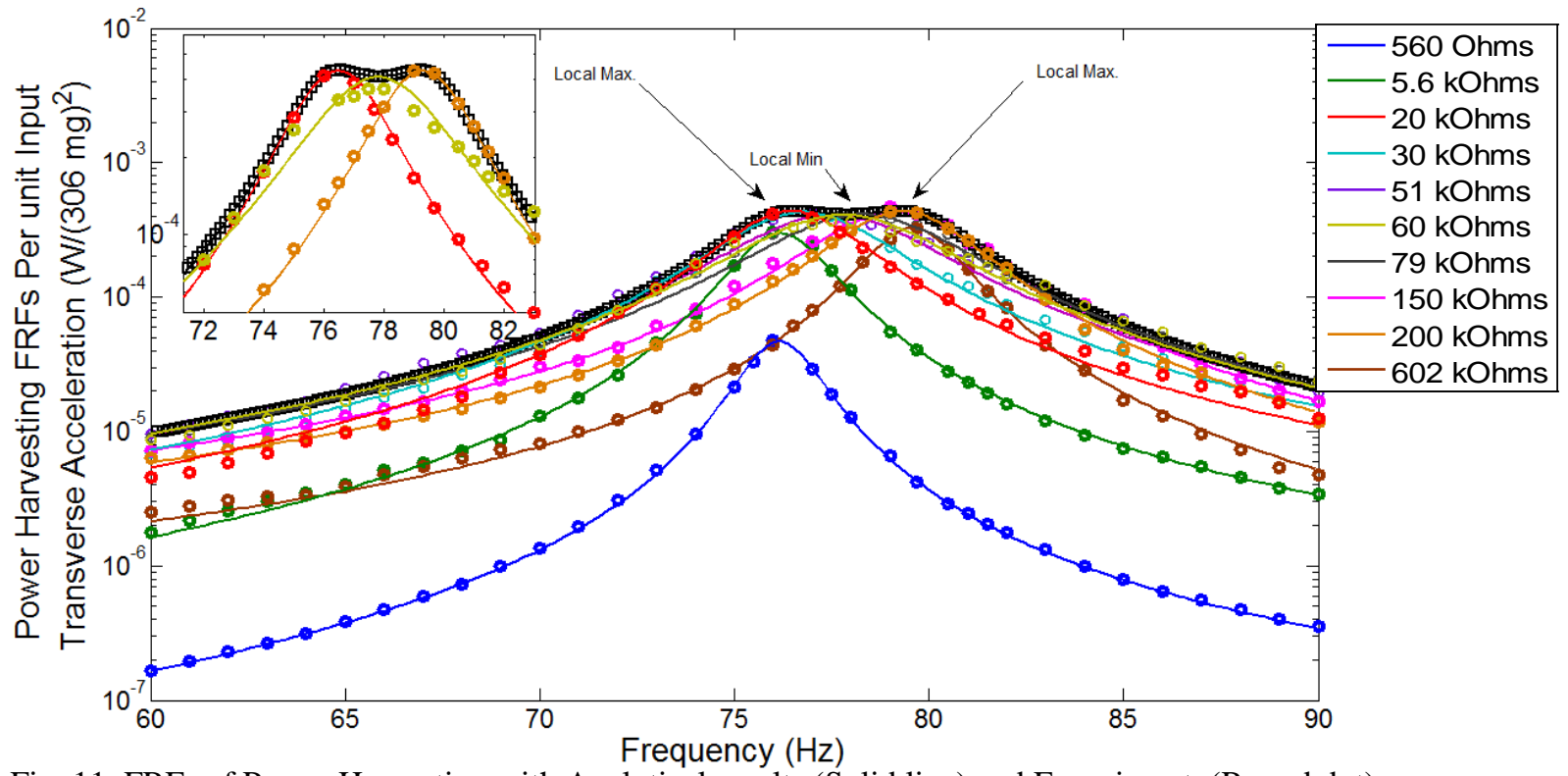

Fig. 11. FRFs of Power Harvesting with Analytical results (Solid line) and Experiment (Round dot) Including Optimal Values From Local Minimum to Maximum (Black Square) 


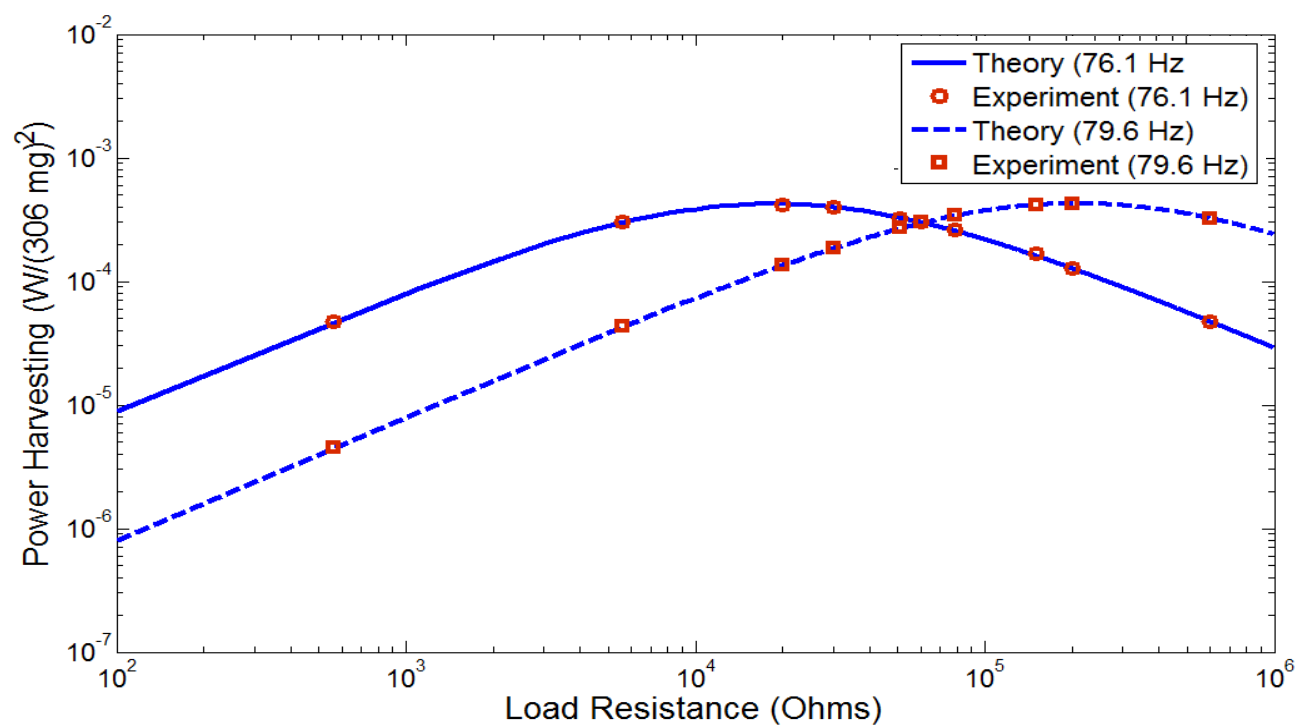

Fig. 12. Power Harvesting versus Load Resistance under the Short circuit Resonance Frequency of $76.1 \mathrm{~Hz}$ and Open Circuit Resonance Frequency of $79.6 \mathrm{~Hz}$
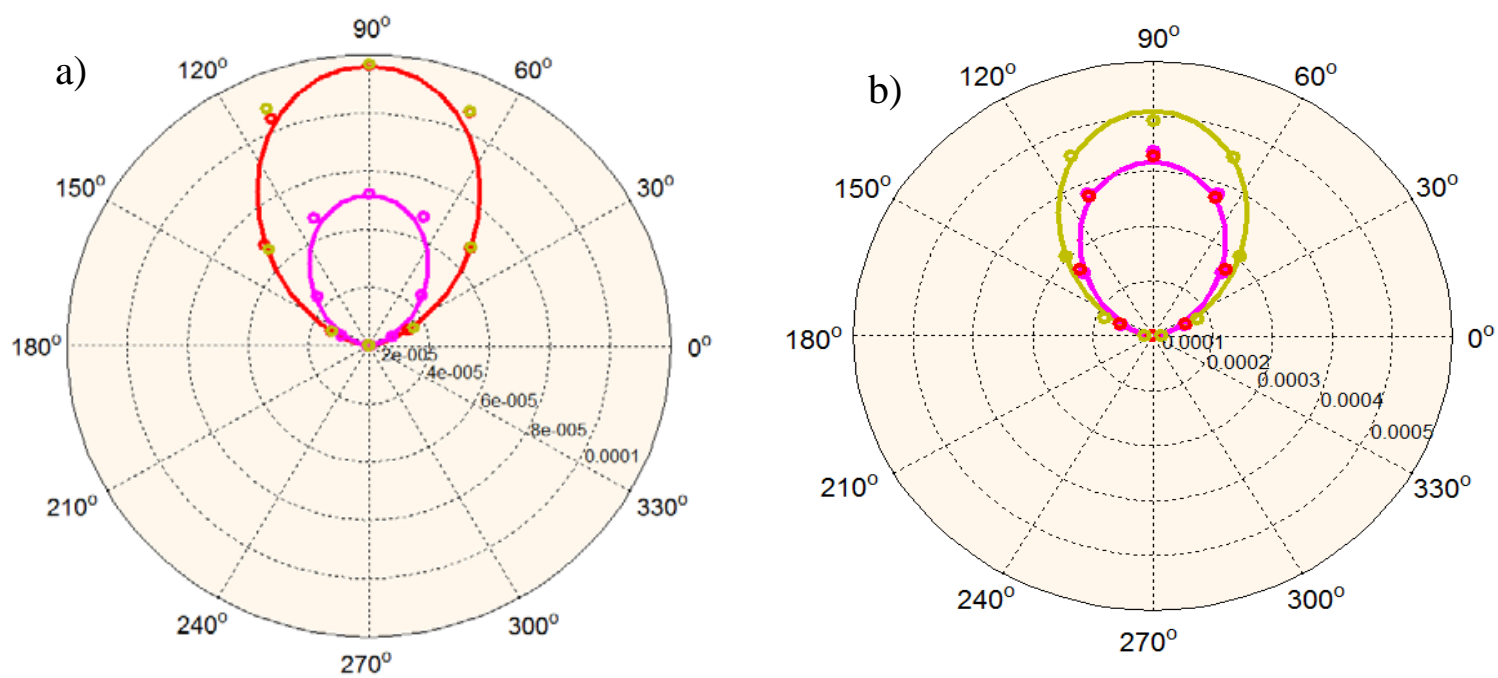

C) $0.23 \mathrm{~mW} /(306 \mathrm{mg})^{2}$
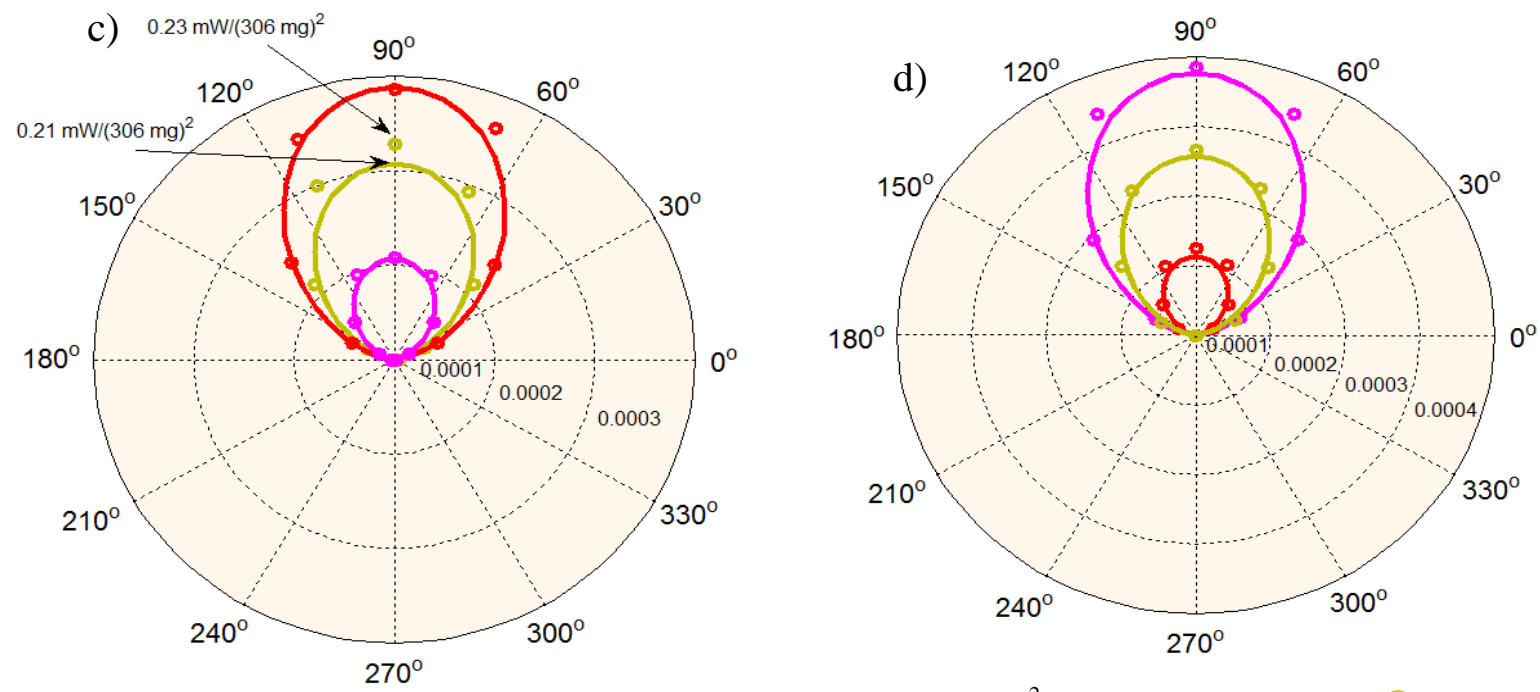

Fig. 13. FRF of Polar Power Harvesting from measurement $\left(\mathrm{W} /(306 \mathrm{mg})^{2}\right)$ at $20 \mathrm{k} \Omega \mathbf{O}$, at $60 \mathrm{k} \Omega \bigcirc$, at $150 \mathrm{k} \Omega \mathbf{O}$ and from theoretical at $20 \mathrm{k} \Omega \longrightarrow$, at $60 \mathrm{k} \Omega \quad$, at $150 \mathrm{k} \Omega$, : (a) $72.67 \mathrm{~Hz}$ (b) $77.71 \mathrm{~Hz}$ (c) $75 \mathrm{~Hz}$ and (d) $80 \mathrm{~Hz}$

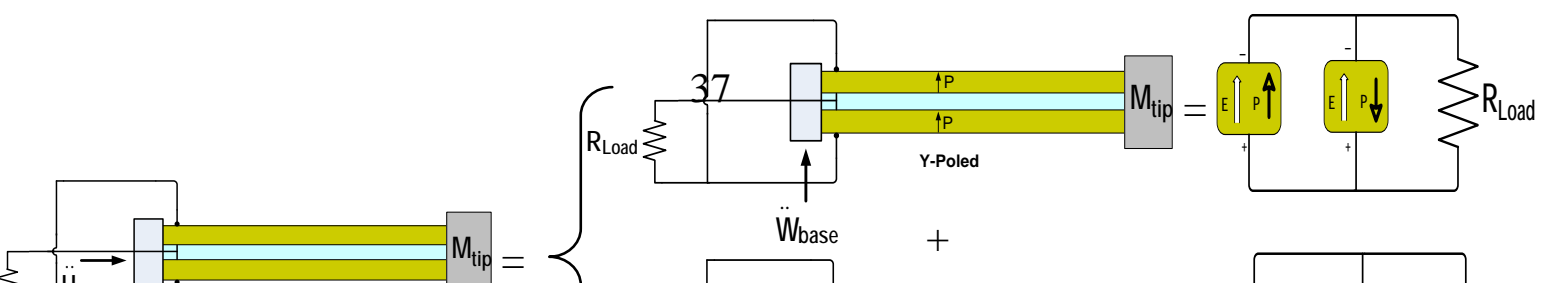


Fig. C.1. Cantilevered piezoelectric bimorph beam with two input base longitudinal and transverse excitations under electrical parallel connections. 